\title{
Calcium-Activated Calpain Specifically Cleaves Glutamate Receptor IIA But Not IIB at the Drosophila Neuromuscular Junction
}

\author{
๑Elsayed Metwally, ${ }^{1,2 *}$ Guoli Zhao, ${ }^{1 *}$ Wenhua Li, ${ }^{1}$ Qifu Wang, ${ }^{1}$ and $\odot$ Yong Q. Zhang ${ }^{1,2}$ \\ ${ }^{1}$ State Key Laboratory for Molecular and Developmental Biology, CAS Center for Excellence in Brain Science and Intelligence Technology, Institute of \\ Genetics and Developmental Biology, Chinese Academy of Sciences, Beijing 100101, China, and 2International College, University of Chinese Academy of \\ Sciences, Beijing 10080, China
}

\begin{abstract}
Calpains are calcium-dependent, cytosolic proteinases active at neutral $\mathrm{pH}$. They do not degrade but cleave substrates at limited sites. Calpains are implicated in various pathologies, such as ischemia, injuries, muscular dystrophy, and neurodegeneration. Despite so, the physiological function of calpains remains to be clearly defined. Using the neuromuscular junction of Drosophila of both sexes as a model, we performed RNAi screening and uncovered that calpains negatively regulated protein levels of the glutamate receptor GluRIIA but not GluRIIB. We then showed that calpains enrich at the postsynaptic area, and the calcium-dependent activation of calpains induced cleavage of GluRIIA at Q788 of its C terminus. Further genetic and biochemical experiments revealed that different calpains genetically and physically interact to form a protein complex. The protein complex was required for the proteinase activation to downregulate GluRIIA. Our data provide a novel insight into the mechanisms by which different calpains act together as a complex to specifically control GluRIIA levels and consequently synaptic function.
\end{abstract}

Key words: calcium; calpain; cytoplasmic cysteine protease; Drosophila neuromuscular junction; GluR; glutamate receptor

\section{Significance Statement}

Calpain has been implicated in neural insults and neurodegeneration. However, the physiological function of calpains in the nervous system remains to be defined. Here, we show that calpain enriches at the postsynaptic area and negatively and specifically regulates GluRIIA, but not IIB, level during development. Calcium-dependent activation of calpain cleaves GluRIIA at Q788 of its $\mathrm{C}$ terminus. Different calpains constitute an active protease complex to cleave its target. This study reveals a critical role of calpains during development to specifically cleave GluRIIA at synapses and consequently regulate synaptic function.

\section{Introduction}

Calpains are a family of calcium-activated cytoplasmic cysteine proteases, which are ubiquitously expressed in various mamma-

\footnotetext{
Received July 31, 2017; revised Dec. 21, 2018; accepted Jan. 16, 2019.

Author contributions: E.M., G.Z., and Y.Q.Z. designed research; E.M., G.Z., W.L., and Q.W. performed research; E.M., G.Z., and Y.Q.Z. analyzed data and wrote the paper.

This work was supported by Strategic Priority Research Program B of the Chinese Academy of Sciences XDBS1020100 and the National Science Foundation of China NSFC 31110103907 and 31490590 to Y.Q.Z. and National Science Foundation of China NSFC Grant 31500824 to G.Z. We thank Drs. Endre Kókai and Helena Araujo for antibodies and flies; Bloomington and Tsinghua stock centers for supplying stocks; Drs. Yingchun Wang and Cathrine Rein Carlson for identification of cleavage sites; Dr. Thomas Schwarz for discussion and advice; and members of the Y.Q.Z. laboratory for discussion and suggestions.

The authors declare no competing financial interests.

*E.M. and G.Z. contributed equally to this work.

Correspondence should be addressed to Yong Q. Zhang at yqzhang@genetics.ac.cn.

E. Metwally's present address: Department of Cytology and Histology, Faculty of Veterinary Medicine, Suez Canal University, Ismailia 41522, Egypt.

G. Zhao's present address: F. M. Kirby Neurobiology Center, Boston Children's Hospital, Boston MA 02115, and Department of Neurobiology, Harvard Medical School, Harvard University, Cambridge MA 02138.
}

lian tissues and are functionally active at neutral $\mathrm{pH}$ (Franco and Huttenlocher, 2005; Hanna et al., 2008). Members of the calpain family act in pathological processes associated with calcium overload, such as ischemia and Alzheimer's disease (Lee et al., 2000; Bano et al., 2005; Xu et al., 2007; Vosler et al., 2011). For example, calpains cleave multiple synaptic proteins, including both inotropic and metabotropic glutamate receptors in excitotoxic conditions, as well as in synaptic plasticity (Chan and Mattson, 1999; Xu et al., 2007; Doshi and Lynch, 2009; Baudry and Bi, 2016). However, little is known of the precise physiological function of calpain-dependent cleavage of target proteins at synapses during normal development.

Calpains exist in organisms ranging from bacteria to humans (Sorimachi et al., 2011; Ono et al., 2016). To date, 15 different 
calpains have been identified in mammals and four in Drosophila (Friedrich et al., 2004; Sorimachi et al., 2011). Unlike most proteases, calpains do not destroy but cleave their substrates at limited sites to modulate their function. Calpain 1 ( $\mu$-calpain) and calpain 2 (m-calpain) are the most ubiquitous and well-studied calpain family members. They are activated in vitro by micromolar and millimolar concentrations of calcium, respectively (Sorimachi et al., 2011; Ono et al., 2016). Conventional calpains 1 and 2 are heterodimers composed of a large catalytic subunit with four domains (dI-dIV) and a small regulatory subunit with two domains ( $\mathrm{dV}$ and $\mathrm{dVI}$ ). Heterodimerization of the large and small subunits occurs through a unique interaction between their C-terminal domains (Strobl et al., 2000; Sorimachi et al., 2011). There are two calcium-binding sites within the crystal structure of the enzymatically active domain II of $\mu$-calpain (Moldoveanu et al., 2002). These sites, together with domains IV and VI, interact with calcium and are required for full enzymatic activity. Calpastatin is an endogenous calpain inhibitor that binds and inhibits calpains via its calpain-inhibitor domains when the proteases are activated by calcium (Hanna et al., 2008). Unlike mammalian calpains, Drosophila calpains comprise a large subunit only and calpastatin has not been identified in Drosophila (Friedrich et al., 2004).

The efficiency of neurotransmission is determined by the level of neurotransmitter receptors at the postsynaptic densities (PSDs). However, the molecular mechanism by which the level of neurotransmitter receptors at synapses is regulated is not well understood. At Drosophila neuromuscular junction (NMJ) synapses, there are two subtypes of glutamate receptors (GluR), GluRIIA and GluRIIB, which are developmentally, biophysically, and pharmacologically distinct (DiAntonio et al., 1999; Marrus et al., 2004; Schmid et al., 2008). For example, the postsynaptic sensitivity to glutamate is much higher at synapses enriched for GluRIIA than at synapses enriched for GluRIIB (Schmid et al., 2008). Previous studies show that GluRIIA and GluRIIB receptors are differentially regulated via independent pathways (Davis et al., 1998; Albin and Davis, 2004; Chen et al., 2005), although the molecular mechanisms that control different subtypes of GluRs remain elusive. To uncover new pathways that control GluR expression at NMJ synapses, we have carried a genetic screen and identified a critical role for calpains in specifically regulating the abundance of GluRIIA, but not GluRIIB, at the Drosophila NMJs. We show that calpains enrich at the postsynaptic area and cleave GluRIIA, but not GluRIIB, at its C-terminal in a calcium-dependent manner. As calcium is a highly versatile intracellular signal that regulates many different cellular functions, our finding of calpain proteinases activated by calcium under physiological conditions at NMJ synapses offers novel insights into the myriad of calcium-mediated processes at other cellular contexts.

\section{Materials and Methods}

Drosophila stocks and genetics. All fly stocks (both males and females) were maintained on standard cornmeal food at $25^{\circ} \mathrm{C}$. All flies were obtained from Bloomington Drosophila Stock Center (http://flystocks.bio. indiana.edu) or Tsinghua University Drosophila Stock Center (China, http://fly.redbux.cn/), unless otherwise noted. The $w^{1118}$ strain was used as the WT control in all experiments. Transgenic flies carrying UAS-calp $A$ were generated as follows. The full-length cDNA of calpain A was amplified by PCR from a cDNA prepared from the adult $w^{1118}$ strain and cloned into a pUAST-attB vector (Zhao et al., 2015). The following primers were used (forward: $5^{\prime}$ gaattcatggacgacttgaggggattctt $3^{\prime}$; reverse: $5^{\prime}$ tctagattacgaatatattgtgcgctctatccac $3^{\prime}$ ). The plasmid was injected into flies carrying an attP2 docking site or attP40 (Best Gene). UAS-calpain A-GFP flies were generously provided by Dr. H. Araujo at the Federal University of Rio de Janeiro (Fontenele et al., 2013). GluRIIA-GFP and GluRIIBRFP flies were documented by Schmid et al. (2008), and Myc-tagged GluRIIA (RRID:BDSC_64258) and GluRIIB (RRID:BDSC_64259) flies were reported by Petersen et al. (1997).

Immunohistochemistry. Standard immunocytochemistry was performed as described previously with minor modifications ( $\mathrm{Li}$ et al., 2016). Third instar larvae were dissected in ice-cold calcium-free HL-3 saline, and then fixed in either $100 \%$ methanol on ice for $10 \mathrm{~min}$ (for anti-GluRIIA and anti-GluRIIB) or 4\% PFA for $30 \mathrm{~min}$ (for all other antibodies), followed by washing with $0.2 \%$ Triton X-100 in PBS. The following primary antibodies were used: mouse anti-GluRIIA (1:500; \#8B4D2, Developmental Studies Hybridoma Bank, RRID:AB_528269), rabbit anti-GluRIIB (1:2500; from Dr. Aaron DiAntonio, Washington University, RRID:AB_2568753), Alexa 649-conjugated anti-HRP (1:100, Jackson ImmunoResearch Laboratories, RRID:AB_2340866), rabbit anti-calp A and anti-calp B (1:500, from Dr. Endre Kókai, Department of Medical Chemistry, Faculty of Medicine, University of Debrecen, Debrecen, Hungary, RRID:AB_2569845), mouse anti-Discs large (Dlg, 1:1000, Developmental Studies Hybridoma Bank, RRID:AB_528203), mouse anti-HA (1:100; Sigma-Aldrich, RRID:AB_514505), rabbit antiHis (1:300; Abcam, RRID:AB_443105), rabbit anti-dPAK (1:500; Zipursky laboratory, University of California at Los Angeles), rabbit anti$\alpha$-spectrin and anti- $\beta$-spectrin (1:500; Dubreuil laboratory, University of Illinois), mouse anti-Fasciclin II, mouse anti-Futsch (1:50; Developmental Studies Hybridoma Bank), and mouse anti-GFP (1:300; Santa Cruz Biotechnology, RRID:AB_627695). Alexa 488- or 568-conjugated anti-mouse, anti-rabbit, or anti-rat secondary antibodies (Invitrogen) were used at 1:500. A nuclear dye To-PRO-3 Iodide (Invitrogen) was used at 1:700.

Immunoblotting (IB) and immunoprecipitation (IP). Western blotting was performed as described previously (Zhao et al., 2015). Third instar larvae muscles were homogenized in ice-cold RIPA lysis buffer $(50 \mathrm{~mm}$ Tris- $\mathrm{HCl}, \mathrm{pH} 7.4,150 \mathrm{~mm} \mathrm{NaCl}, 0.1 \%$ SDS, $1 \% \mathrm{NP}-40$ ), with a protease inhibitor (Set 1, Roche Diagnostic). The lysates were centrifuged at $18,000 \times g$ for $10 \mathrm{~min}$, and the resulting supernatant was used for Western blotting with the following primary antibodies: mouse antiGluRIIA (1:1000), rabbit anti-RFP (1:700, Creative Diagnostic, RRID: AB_2433853), rabbit anti-calp A and anti-calp B (1:1000), mouse anti$\beta$-actin and anti- $\alpha$-tubulin (1:50,000, Millipore). The secondary antibody was HRP-conjugated anti-mouse IgG or anti-rabbit IgG (Sigma-Aldrich) used at 1:50,000. Protein bands were visualized using a chemiluminescent HRP substrate (Millipore). For IP, larval muscle lysates were incubated with appropriate antibodies ( $2 \mu \mathrm{g}$ antibody). Protein complexes were precipitated with Dynabeads (Thermo Fisher Scientific) for further analysis.

Calcium treatment of larval muscles. To induce activation of the endogenous calpains, larvae were dissected in $\mathrm{Ca}^{2+}$-free HL-3 saline. For unstimulated control preparations, this saline included $0.5 \mathrm{~mm}$ EGTA. For stimulated preparations, dissected larvae were then incubated with HL-3 solution containing $10 \mathrm{mM} \mathrm{CaCl}_{2}$ and $10 \mu \mathrm{M}$ ionomycin (Sigma-Aldrich, \#19657) for $30 \mathrm{~min}$ and then washed in $\mathrm{Ca}^{2+}$-free HL-3 saline and left at rest for $10 \mathrm{~min}$. For calpain activity inhibition, $10 \mu \mathrm{M}$ PD150606 (Abcam, \#ab141464) was added to the solution for 30 min before the addition of calcium. For immunohistochemistry, differently treated groups were dissected and processed together to ensure equivalent treatments. For Western blotting, lysates from different groups were IPed with anti-Myc $(2 \mu \mathrm{g})$, and the resulting immunoprecipitates were detected with antiMyc (1:1000).

Optogenetic manipulation of calcium influx. For optogenetic manipulation of calcium influx, the following genotypes were used: C57-Gal4/ +;UAS-ChR2/+, C57-Gal4 Calp A RNAi/+;UAS-ChR2/+, and C57Gal4 $\alpha I D$ RNAi/+;UAS-ChR2/+ . Larvae expressing blue light-sensitive channelrhodopsin-2 (ChR2) by C57-Gal4 were kept in consistent darkness and raised in standard food containing $0.1 \mathrm{~mm}$ all-trans-retinal (Sigma-Aldrich, R2500) that is necessary for the production of functional ChR2 (Honjo et al., 2012). Late third instar larvae were subjected to intermittent exposure of blue light of $\sim 480 \mathrm{~nm}$ by a fluorescent microscope. The light illumination protocol included five rounds of $10 \mathrm{~min}$ 
light stimulation followed by a $30 \mathrm{~min}$ break. During the $10 \mathrm{~min}$ illumination period, the light turned on and off for $30 \mathrm{~s}$ each for 10 times. Larvae expressing ChR2 exhibited apparent muscle contractions upon illumination, validating the genotypes.

Cell culture and plasmid construction. Drosophila S2 cells were cultured as previously described (Li et al., 2016). S2 cells (RRID:CVCL_Z232) were maintained at $25^{\circ} \mathrm{C}$ in a serum-free medium (SF-900 II, Invitrogen). Cultured cells were resuspended in the medium at a final concentration of $1 \times 10^{6}$ cells/ml medium and plated at $1 \mathrm{ml} /$ well in six-well culture plates. Plasmids were added after $24 \mathrm{~h}$ growth in the culture plates. Cells expressing exogenous proteins of interest were transfected using Cellfectin II reagent (Invitrogen), and expression was induced with $0.5 \mathrm{mM} \mathrm{CuSO}_{4}$ for $48 \mathrm{~h}$. Cells were lysed in RIPA buffer for $1 \mathrm{~h}$ at $4^{\circ} \mathrm{C}$.

For in vitro GluRIIA cleavage assay, GluRIIA was subcloned into pAC5.1 plasmids to produce N-terminally Flag-tagged fusion proteins. Transfected S2 cells were lysed in RIPA buffer, and the lysates were diluted (1:1) in $2 \times$ calpain buffer $(1 \times$ calpain buffer: $0.1 \%$ Triton, $20 \mathrm{~mm}$ HEPES, pH 7.5) (Bano et al., 2005). After adding $10 \mathrm{~mm} \mathrm{CaCl}_{2}$ to the lysates, samples were kept at $37^{\circ} \mathrm{C}$ for $1 \mathrm{~h}$ for proteolysis to occur. To inhibit calpain activity, lysates were incubated with $10 \mu \mathrm{M}$ PD15606 for $30 \mathrm{~min}$ before the addition of calcium. The reaction was stopped by adding $5 \times$ SDS loading buffer and subjected to Western blotting with anti-Flag (1:1000).

The following fusion proteins were coexpressed in S2 cells: Calp A-His with Calp B-His, Calp C-His, Calp D-His, or Flag-Calp B. For IP, lysates from S2 cells were incubated with specific antibodies $(3 \mu \mathrm{g})$, including rabbit anti-calpain A or mouse anti-Flag and subsequently precipitated with $50 \mu$ l Dynabeads (Invitrogen). An equal amount of rabbit or mouse IgG ( $3 \mu \mathrm{g}$, Sigma-Aldrich) was used as a negative IP control.

Prediction and verification of calpain cleavage sites in GluRIIA. CaMPDB (calpain for modulatory proteolysis database) (DuVerle et al., 2011) and GPS-CCD 1.0 program (calpain cleavage detector) (Liu et al., 2011) were used to identify putative calpain cleavage sites in the peptide from G785 to F840 in the cytoplasmic region of GluRIIA. Both programs predicted two high-score calpain cleavage sites at Q788 and K791. For site-directed mutagenesis, the predicted GluRIIA cleavage sites were mutated with the KOD-Plus-Mutagenesis Kits (KOD-201, F0934K, Toyobo) according to the manufacturer's recommendations. The following primers were used to mutate the predicted sites to alanine for cleavage assay. For Q788A mutation, we used forward primer ( $5^{\prime}$ cgaaaggggcgagctggcgaagatgaagaacaagtggtggaagaacc $3^{\prime}$ ) and reverse primer (5' ggttcttccaccacttgttcttcatcttcgccagctcgcccctttcg $\left.3^{\prime}\right)$. For K791A mutation, we used forward primer ( $5^{\prime}$ cgaaaggggcgagctgcagaagatggcgaacaagtggtggaagaacc $\left.3^{\prime}\right)$ and reverse primer $\left(5^{\prime}\right.$ ggttcttccaccacttgttcgccatcttctgcagctcgccctttcg $3^{\prime}$ ). For Q788A-K791A double mutation, we used forward primer $\left(5^{\prime}\right.$ cgaaaggggcgagctggcgaagatggcgaacaagtggtggaagaacc $\left.3^{\prime}\right)$ and reverse primer (5' ggttcttccaccacttgttcgccatcttcgccagctcgcccctttcg $3^{\prime}$ ). GluRIIA with or without mutations expressed in S2 cells was tested for calpain cleavage. To facilitate calpain cleavage, we treated S2 cells with 4-hydroxy nonenal (HNE) (Calbiochem, \#393204) at various concentrations.

Electrophysiology. Intracellular recordings from Drosophila muscles were performed essentially as described previously (Zhao et al., 2015). Briefly, wandering third instar larvae were dissected in calcium free HL-3 saline (70 mм NaCl, $5 \mathrm{~mm} \mathrm{KCl,} 20 \mathrm{~mm} \mathrm{MgCl}_{2}, 10 \mathrm{~mm} \mathrm{NaHCO}_{3}, 5 \mathrm{~mm}$ trehalose, $5 \mathrm{~mm}$ HEPES, $115 \mathrm{~mm}$ sucrose, $\mathrm{pH}$ 7.3). Excitatory junction potentials (EJPs) and miniature EJPs (mEJPs) were recorded from muscle 6 of abdominal segment A2 or A3 in HL-3 saline containing $0.5 \mathrm{~mm}$ $\mathrm{Ca}^{2+}$ using intracellular microelectrodes $(10-20 \mathrm{M} \Omega$ ) filled with $3 \mathrm{M} \mathrm{KCl}$, and processed with Clampfit 10.2 software. All electrophysiological responses were recorded using an Axoclamp 2B amplifier (Molecular Devices) in Bridge mode and analyzed using Clampfit 10.2 software. Only recordings from cells with resting potentials $\leq-60 \mathrm{mV}$ and input resistances $>6 \mathrm{M} \Omega$ were analyzed.

Image acquisition and data analysis. Images were collected using an Olympus Fluoview FV1000 confocal microscope with a $40 \times / 3$ NA or $60 \times / 3$ NA oil objective and FV10-ASW software (RRID:SCR_014215) or with a confocal microscope (Leica Microsystems) using a $40 \times / 3$ NA oil objective and LAS AF software (RRID:SCR_013673). All images of mus- cle 4 Type Ib NMJs for any specific experiment were captured using identical settings to allow for statistical comparisons among different genotypes. Immunostaining intensities were quantified using ImageJ (National Institutes of Health, RRID:SCR_003070), with anti-HRP staining as an internal control for quantification. An arbitrary threshold was set for each channel and used for all relevant images. The average intensity of GluR signal within the ROI defined by HRP staining was normalized to the average of HRP intensity. To quantify protein levels of target proteins, positive signals on Western blots from multiple independent repeats were calculated using ImageJ and normalized to the loading control. Each experiment was repeated at least three times. Statistical comparisons were performed using GraphPad Prism 6. All data are expressed as mean \pm SEM.

Statistical analyses. Different groups were compared by one-way ANOVA with Tukey's post hoc tests for pairwise comparisons or Student's $t$ tests when appropriate. In all figures, data are mean \pm SEM. The exact $p$ values, $F$ values, $t$ values, and coefficient of determination $\left(R^{2}\right)$ of statistical analysis are reported. All comparisons were between a specific genotype and the control unless otherwise indicated.

\section{Results \\ Calpains negatively regulate synaptic and total GluRIIA protein levels}

To elucidate the molecular pathways that control synaptic expression of GluRs, we screened 500 selected RNAi lines (one line was tested for one gene) under the control of the muscle-specific C57-Gal4. We used GFP intensity of the GluRIIA-tagged with GFP at its $C$ terminus as a readout for the screening. GluRIIAGFP was confined to individual PSDs and functioned as the endogenous GluRIIA (Qin et al., 2005; Schmid et al., 2008). From the 500 RNAi lines tested, we identified 18 lines (3.6\%) associated with significant upregulation of GluRIIA at NMJ terminals (Table 1-1, available at https://doi.org/10.1523/JNEUROSCI.221317.2019.t1-1). Among these 18 genes, we focused on the calciumdependent protease calpain. Four distinct genes encoding calpains have been described in Drosophila, although the calpain $\mathrm{C}$ might not have proteolytic activity as the active site residues are mutated (Friedrich et al., 2004).

We verified the screening results by examining synaptic GluRIIA levels in multiple calpain mutants carrying transposon insertions ( $\operatorname{calp} A^{\text {KG13868 }}$, calp $B^{\text {EY17422 }}$, and calp $D^{\text {EY02271 }}$ ); of these mutants, calp $A^{K G 13868}$ and calp $B^{\text {EY17422 express reduced levels of }}$ calpain protein and proteinase activity (Reinecke et al., 2011). Consistently, homozygous mutants for calp $A, B$, and $D$ showed increased abundance of GluRIIA, but not GluRIIB, at NMJ synapses (Fig. 1A). In support of the staining results, Western blotting showed a significant threefold increase in the total protein level of GluRIIA in different calpain mutants. Compared with WT control, the relative GluRIIA intensity was $320 \pm 4.5$ for Calp $A^{K G}(p=0.00005), 300 \pm 5.3$ for Calp $B^{E Y}(p=0.00002)$, and $320 \pm 5.5$ for calp $D^{E Y}(p=0.00006)\left(F_{(3,8)}=148.3, R^{2}=0.9820\right.$, ANOVA; Fig. $1 B, D)$. To determine the specificity of calpain for GluRIIA, we performed immunoblotting analysis of red fluorescent protein (RFP)-tagged GluRIIB (Schmid et al., 2008) with anti-RFP (antibody against GluRIIB for Western analysis was not available), and showed that GluRIIB-RFP levels were unaltered by calpain A and B knockdown. Compared with control, the relative GluRIIB intensity was $100 \pm 7.5$ for Calp $A^{R N A i}(p=$ $0.9709)$ and $100 \pm 5.6$ for Calp $B^{R N A i}(p=0.9716)\left(F_{(2,6)}=\right.$ $0.0297, R^{2}=0.0098$, ANOVA; Fig. $\left.1 C, D\right)$, demonstrating the specificity of calpain on GluRIIA.

We determined whether calpain was sufficient to cleave GluRIIA in vivo by using the Gal4/UAS system to overexpress calpain A. Synaptic and total GluRIIA levels were significantly decreased when calpain A was overexpressed by C57-Gal4 (Fig. 
A
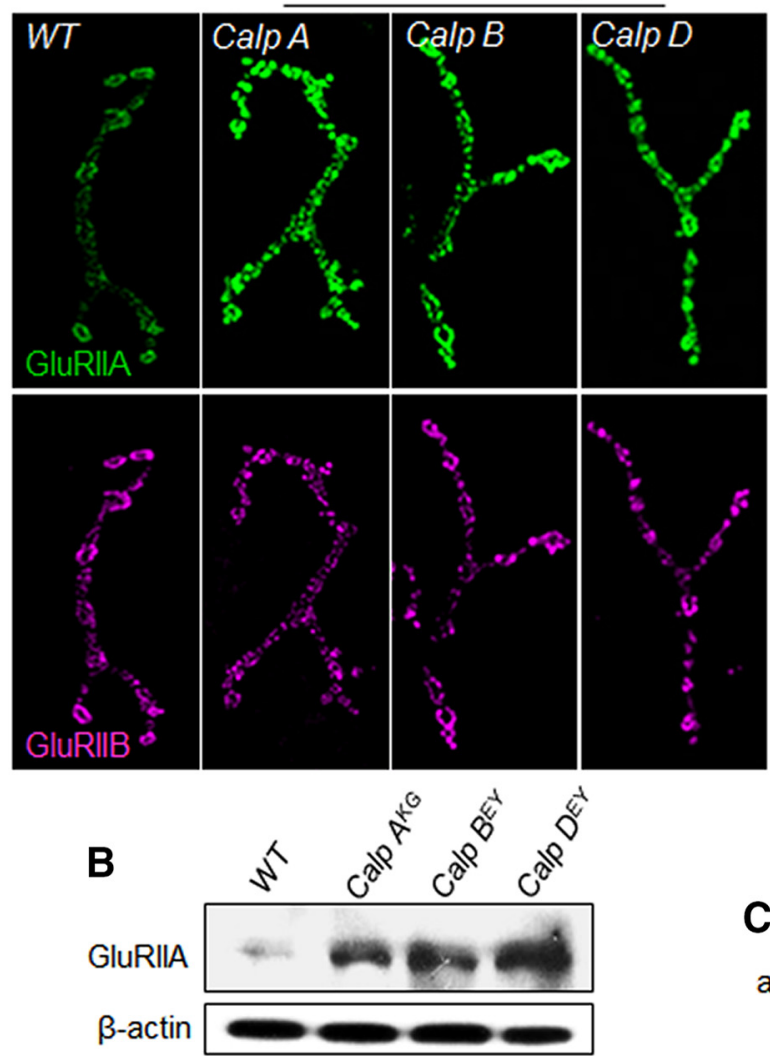

D

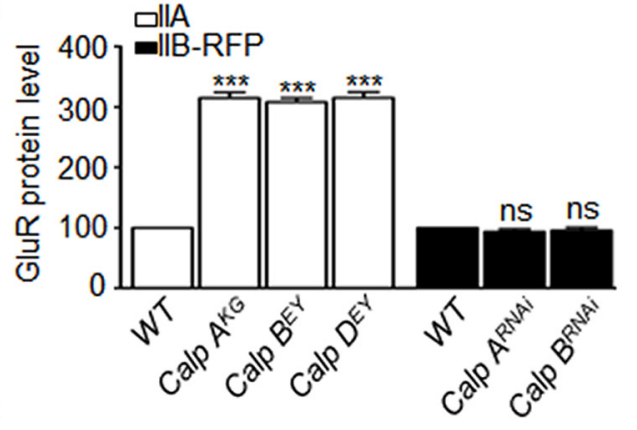

$\mathbf{E}$
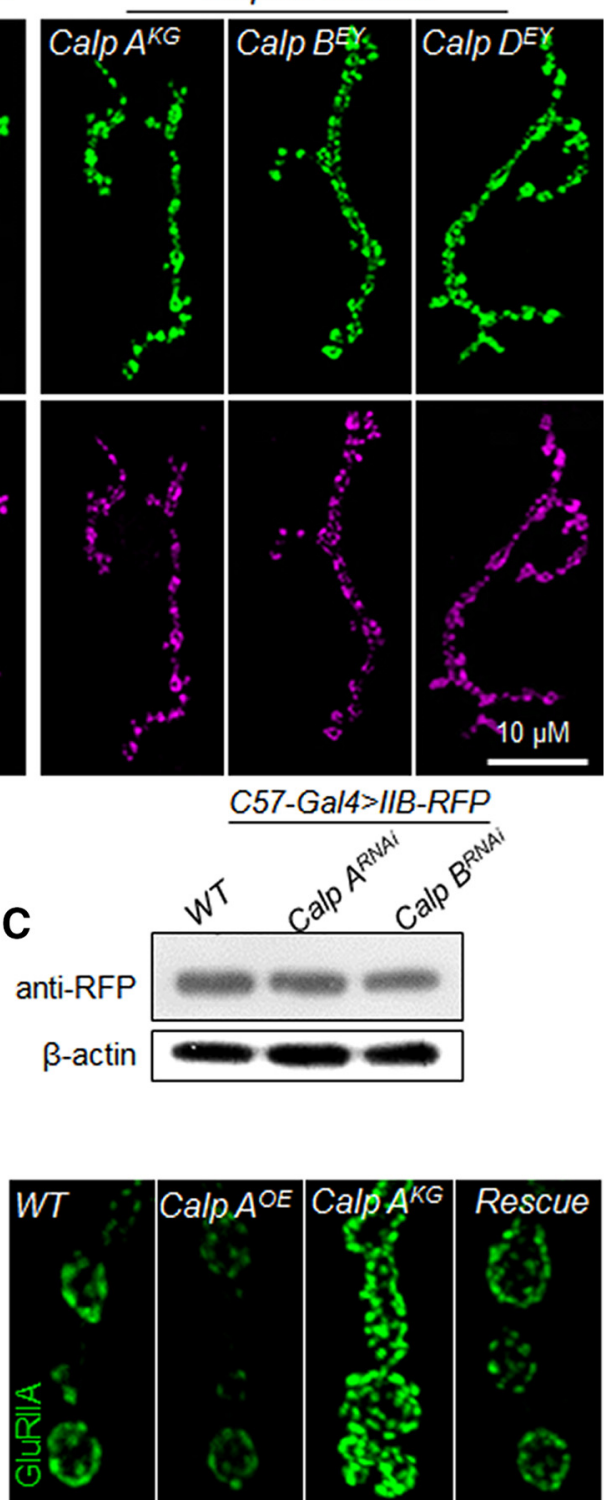

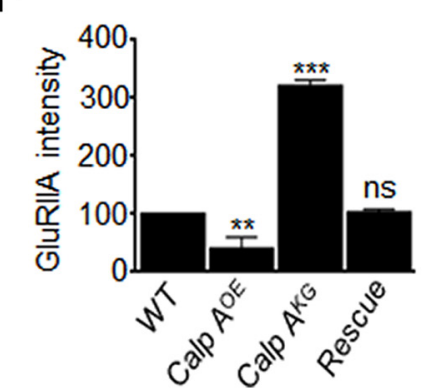

G $\quad$ C57-Gal4

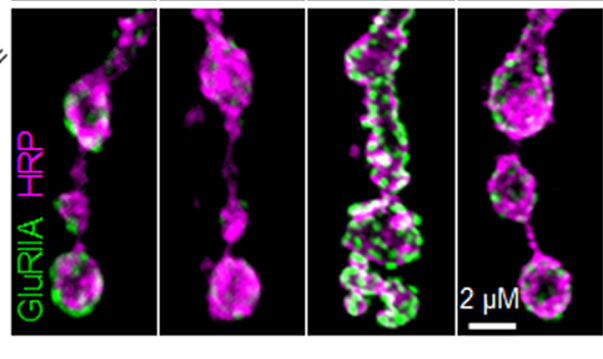

Figure 1. Calpains negatively regulate synaptic and total levels of GluRIIA, but not GluRIIB, protein. A, Synaptic levels of GluRIIA, but not IIB, were increased in both calpain mutants and postsynaptic RNAi knockdown larvae. Representative images of NMJ4 synapse from different genotypes double-stained with anti-GluRIIA (green) and anti-GluRIIB (magenta). Scale bar, $10 \mu \mathrm{m}$. For a list of positive RNAi lines identified for increased GluRIIA expression at NMJ, see also Table 1-1 (available at https://doi.org/10.1523/JNEUROSCI.2213-17.2019.t1-1). $B$, Total GluRIIA protein levels were increased in calpain mutants. Representative immunoblots detected with anti-GluRIIA in different genotypes. $\beta$-Actin was used as a loading control. C, Representative immunoblots showing RFP-tagged GluRIIB levels in different genotypes. $\beta$-Actin was used as a loading control. D, Quantitative analysis of total GluRIIA and GluRIIB-RFP protein levels. $n=3 .{ }^{* * *} p<0.001$ (one-way ANOVA with Tukey's post hoc test). Data are mean \pm SEM. $E$, Calpain A overexpression reversed synaptic GluRIIIA levels in calpain A mutants. Representative images of NMJ4 synapse from WT, calp $A^{O E}$ (UAS-calp A/C57-Gal4), calp $A^{K G}$, and rescue (calp $A^{K G}$; UAS-calp A/C57-Gal4) costained with anti-GluRIII (green) and anti-HRP, a neuronal membrane marker (magenta). Scale bar, $2 \mu \mathrm{m}$. $\boldsymbol{F}$, Quantitative analysis of fluorescence intensity of synaptic GluRIIIA levels from different genotypes. $n=15 \mathrm{NMJs} .{ }^{* *} p<$ $0.01 ;{ }^{* * *} p<0.001$; one-way ANOVA with Tukey's post hoc test. Data are mean \pm SEM. G, Representative immunoblots showing total GluRIIA and calpain A protein levels from different genotypes. $\alpha$-Tubulin was used as a loading control. 
A

\begin{tabular}{|c|c|c|c|r|c|}
\hline Egg & Embryo & $\begin{array}{c}1^{\text {st }} \text { Instar } \\
\text { Larva }\end{array}$ & $\begin{array}{c}2^{\text {nd }} \text { Instar } \\
\text { Larva }\end{array}$ & $\begin{array}{c}3^{\text {rd }} \text { Instar } \\
\text { Larva }\end{array}$ & $\begin{array}{c}\text { GluRIIA } \\
\text { level }\end{array}$ \\
\hline $12 \mathrm{~h}$ & $10 \mathrm{~d} / 18^{\circ} \mathrm{C}$ & & \\
\hline $12 \mathrm{~h}$ & $8 \mathrm{~d}+18 \mathrm{~h}$ & $6 \mathrm{~h}$ & $\mathrm{~ns}$ \\
\hline $12 \mathrm{~h}$ & $8 \mathrm{~d}+12 \mathrm{~h}$ & $12 \mathrm{~h}$ & \\
\hline $12 \mathrm{~h}$ & $8 \mathrm{~d}+6 \mathrm{~h}$ & $1 \mathrm{~d}$ & $*$ \\
\hline $12 \mathrm{~h}$ & $8 \mathrm{~d}$ & $2 \mathrm{~d}$ & $* *$ \\
\hline $12 \mathrm{~h}$ & $6 \mathrm{~d}$ & $3 \mathrm{~d}$ & ${ }^{* * *}$ \\
\hline $12 \mathrm{~h}$ & $4 \mathrm{~d}$ & & \\
$12 \mathrm{~h}$ & $5 \mathrm{~d} / 30^{\circ} \mathrm{C}$ & & \\
\hline
\end{tabular}

B
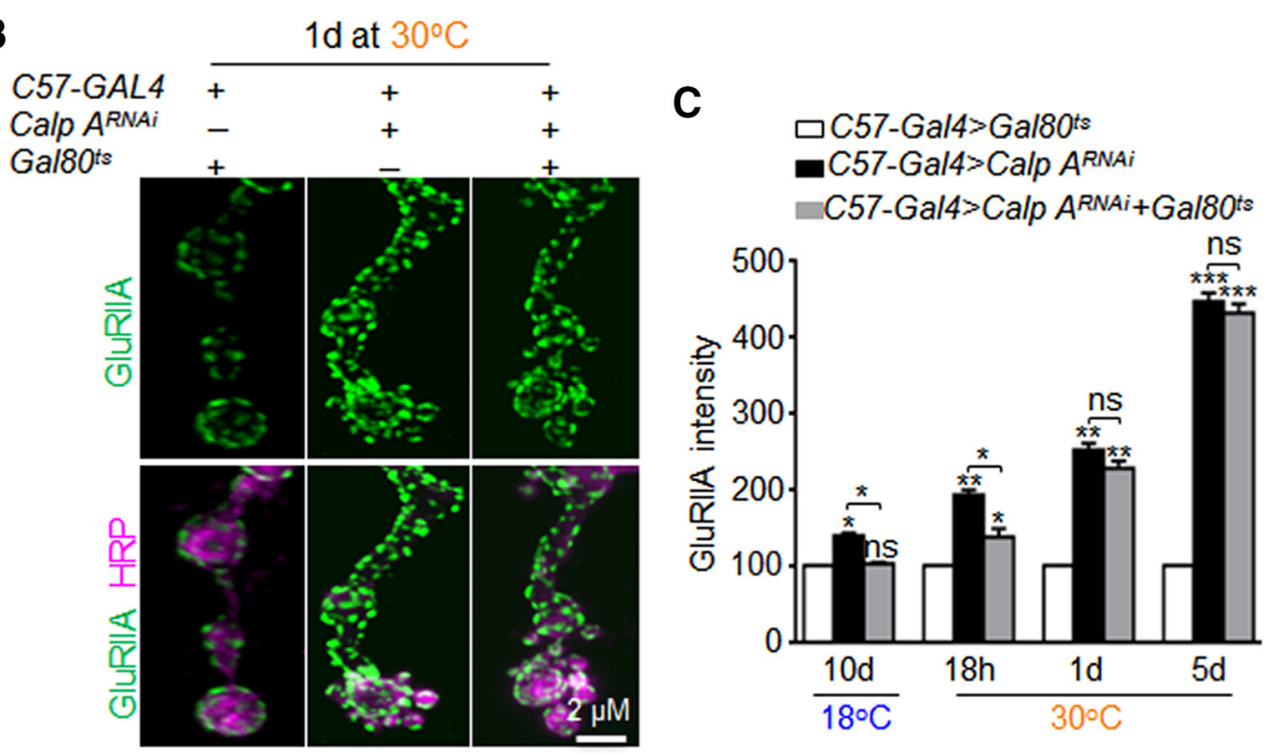

Figure 2. Temperature-sensitive temporal calpain inactivation leads to upregulation of GluRIIA at NMJ. $\boldsymbol{A}$, Table of GluRIIA levels at NMJ synapses of $\left(57-G a l 4>\right.$ Calp $A^{R N A i}+G$ al $80^{t s}$ after temperature shift from $18^{\circ} \mathrm{C}$ (blue) to $30^{\circ} \mathrm{C}$ (orange). ${ }^{*} p<0.1 ;^{* *} p<0.01$; ${ }^{* * *} p<0.001$; one-way ANOVA with Tukey's post hoc test. $\boldsymbol{B}$, Representative images of NMJ4 synapse from different genotypes after temperature shift from $18^{\circ} \mathrm{C}$ to $30^{\circ} \mathrm{C}$ for $1 \mathrm{~d}$ double-stained with anti-GluRIIA (green) and anti-HRP (magenta). Scale bar, $2 \mu \mathrm{m}$. $\mathrm{C}, \mathrm{Quantitative}$ analysis of fluorescence intensity of

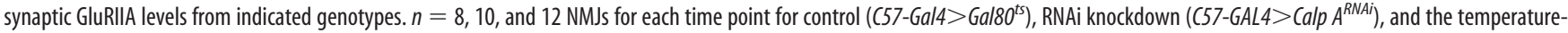
sensitive genotype $\left(C 57-G a l 4>\right.$ Calp $\left.A^{R N A i}+G a l 80^{t 5}\right)$, respectively. ${ }^{*} p<0.1 ;{ }^{* *} p<0.01 ;{ }^{* *} p<0.001 ;$ one-way ANOVA with Tukey's post hoc test. Data are mean \pm SEM.

$1 E-G)$. Moreover, overexpression of calpain A in postsynaptic muscles fully rescued the increased GluRIIA phenotype in calpain $A$ mutants. The relative intensity of GluRIIA was $46 \pm 2.5$ for Calp $A^{O E}(p=0.0013), 320 \pm 4.9$ for Calp $A^{K G}(p=0.00004)$, and $100 \pm 6.1(p=0.3254)$ for the rescue compared with WT $\left(F_{(3,56)}=2253, R^{2}=0.9918\right.$, ANOVA; Fig. $\left.1 E, F\right)$. Together, these results demonstrate that calpain $A$ is both required and sufficient to downregulate GluRIIA.

Temperature-sensitive temporal inactivation of calpain leads to upregulation of GluRIIA at NMJ

In the results shown above, calpain activity was chronically downregulated or upregulated from embryonic stage. As it is not obvious that eliminating calpain directly leads to the large increase in GluRIIA, we examined the time course of the increase in GluRIIA elicited by calpain RNAi by using the temperaturesensitive Gal80 ${ }^{t s}$ system (McGuire et al., 2003). When embryos and larvae were raised at $18^{\circ} \mathrm{C}, \mathrm{Gal} 80^{\text {ts }}$ suppressed Gal4, blocking calpain RNAi expression, and GluRIIA remained normal. Compared with the control of $C 57-G a l 4>G a l 80^{t s}$, the relative GluRIIA intensity was $130 \pm 3.4$ for C57-Gal4 $>$ Calp $A^{R N A i}(p=$ $0.0092)$ and $100 \pm 5.5$ for C57-Gal4>Calp $A^{R N A i}+G a l 80^{t s}(p=$ $0.1223)\left(F_{(2,27)}=5.6\right.$, ANOVA; Fig. $\left.2 A, C\right)$. RNAi knockdown of calpain A activity for $18 \mathrm{~h}$, but not for 6 or $12 \mathrm{~h}$, induced a slight but significant upregulation of GluRIIA (Fig. $2 A, C$ ), whereas a longer time of calpain inactivation at $30^{\circ} \mathrm{C}(1-5 \mathrm{~d})$ upregulated GluRIIA levels to a greater extent at NMJ synapses. The relative GluRIIA intensity of C57-Gal4> Calp $A^{R N A i}+$ Gal80 $0^{t s}$ was $130 \pm$ 2.1 at $18 \mathrm{~h}(p=0.0098), 230 \pm 3.2$ at $1 \mathrm{~d}(p=0.0012)$, and $430 \pm$ 5.1 at $5 \mathrm{~d}(p=0.00005)$ after temperature shift $\left(F_{(3,44)}=369.3\right.$, $R^{2}=0.9599$, ANOVA; Fig. $\left.2 A-C\right)$. Moreover, both C57Gal4 $>$ Calp $A^{R N A i}$ and C57-Gal4>Calp $A^{R N A i}+$ Gal80 $0^{t s}$ showed increased GluRIIA levels when raised at $30^{\circ} \mathrm{C}$ for $18 \mathrm{~h}$ (Fig. $2 \mathrm{C}$ ), although this increase tended to be significantly higher in C57Gal4 $>$ Calp $A^{R N A i}\left(p=0.0126 ; R^{2}=0.7096, t_{(20)}=5.224\right.$, unpaired $t$ test; Fig. $2 C$ ). When raised at $30^{\circ} \mathrm{C}$ for a longer time of 1 or $5 \mathrm{~d}$, we did not observe significant differences between the two genotypes $\left(p=0.5813 ; R^{2}=0.1828, t_{(20)}=1.458\right.$, unpaired $t$ test at $1 \mathrm{~d}$ and $p=0.6772 ; R^{2}=0.1032, t_{(20)}=1.333$, unpaired $t$ test at $5 \mathrm{~d}$; Figure $2 C$ ), probably due to similar levels of calpain knockdown during larval development. Together, these data reveal that temporal calpain inactivation induced an increase in GluRIIA abundance at NMJ synapses.

\section{Calpain A is enriched at the postsynaptic NMJ}

Previous studies have shown that human calpains 1 and 2 are ubiquitously expressed (Hood et al., 2004; Cao et al., 2007; Baudry and Bi, 2016). To examine whether calpains were local- 

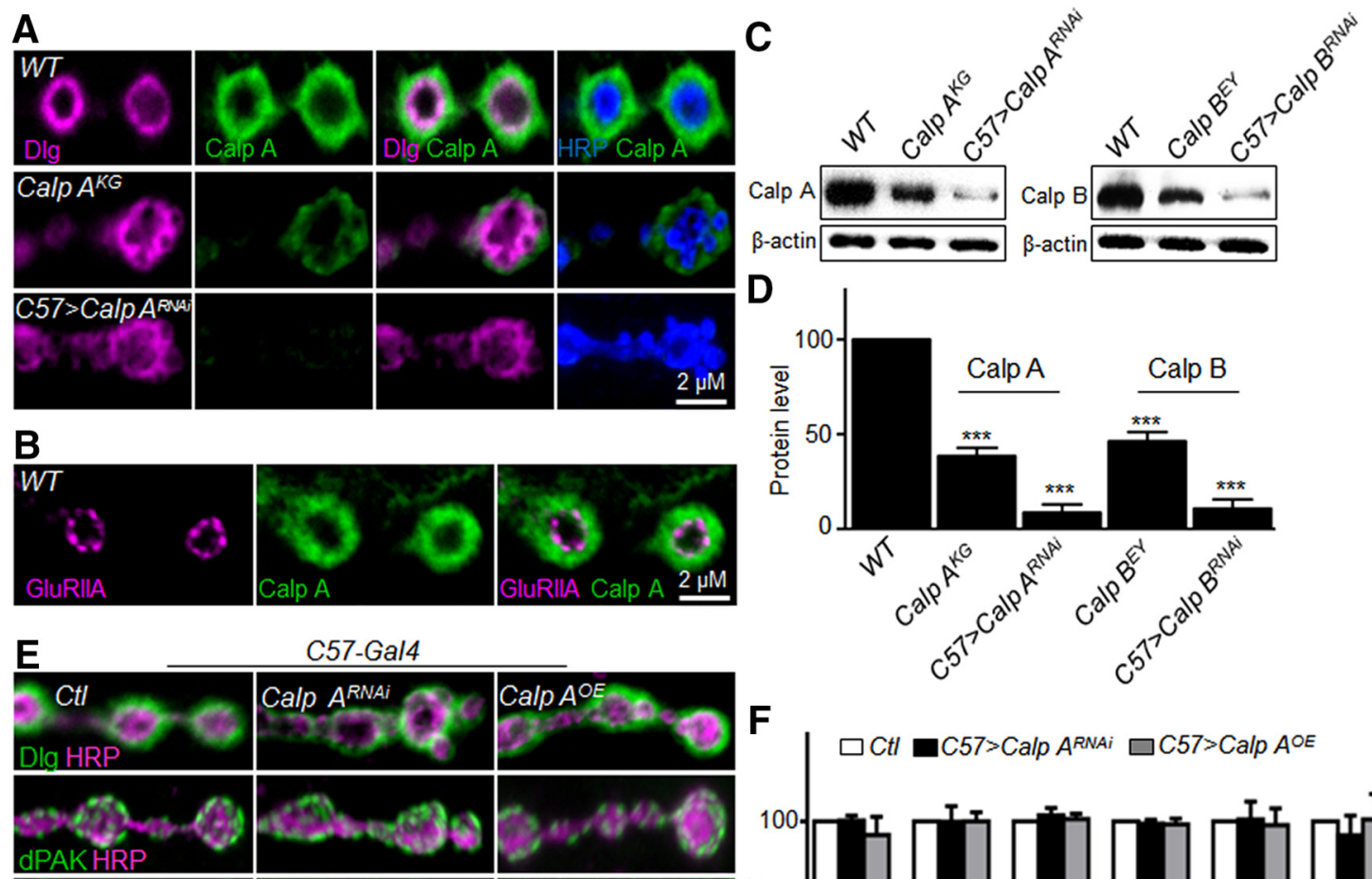

C57-Gal4
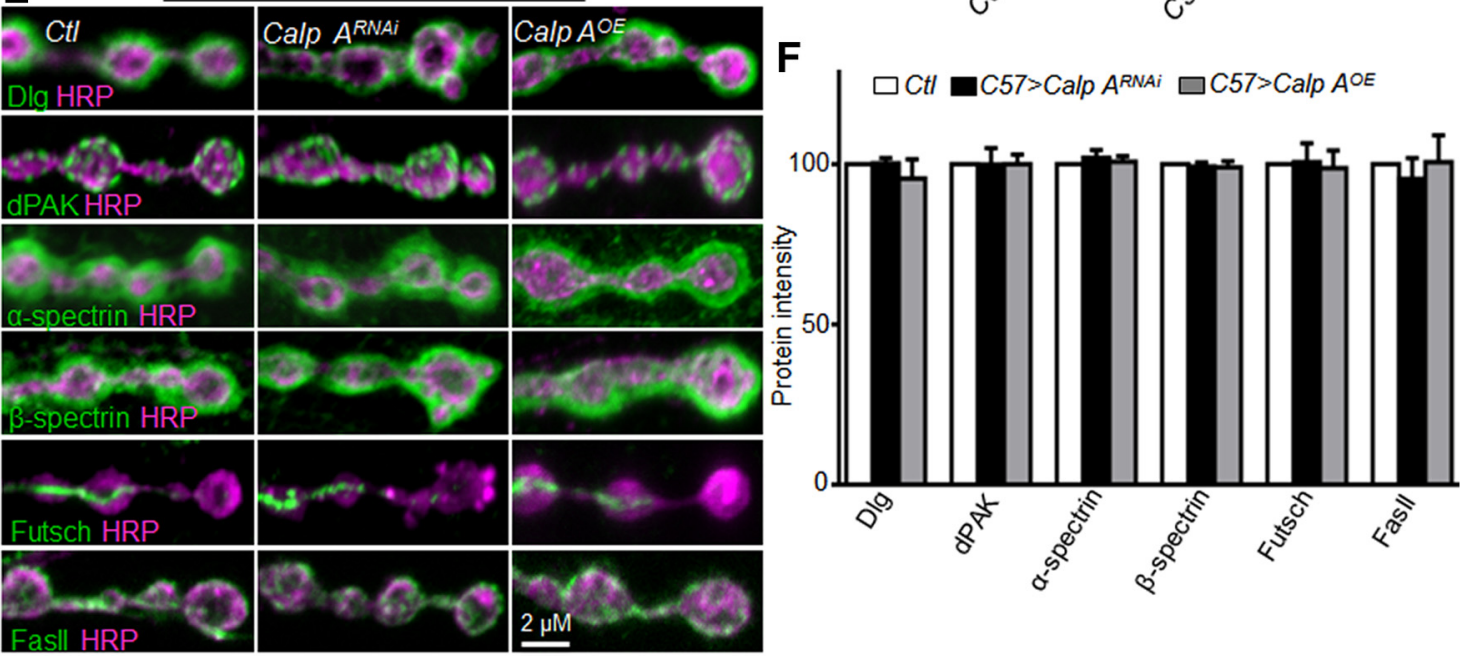

Figure 3. Calpain A enriches at postsynaptic NMJ.A, Calpain A enriches at postsynaptic NMJ. Representative images of NMJ4 synapses from different genotypes, stained with anti-Dlg (magenta), anti-Calp A (green), and anti-HRP (blue). Scale bar, $2 \mu \mathrm{m}$. B, Representative images of WT NMJ4 synapse, stained with anti-GluRIIIA (magenta) and anti-calp A (green). Scale bar, $2 \mu \mathrm{m}$. C, Representative immunoblots probed with anti-calp $A$ and anti-calp B showing calpain protein level in different genotypes. $\beta$-Actin was used as a loading control. $D$, Quantitative analysis of the total protein level of calpains in calpain mutants and RNAi knockdown larvae. $n=3 .{ }^{* * *} p<0.001$ (one-way ANOVA with Tukey post hoc test). Data are mean \pm SEM. $E$, A set of synaptic proteins remain unchanged at NMJs when calpain A is knocked down or overexpressed in the postsynaptic muscles. Representative images of NMJ4 synapses from indicated genotypes double-stained with antibodies against indicated synaptic markers. The intensities of DLG, dPAK, $\alpha$-spectrin, $\beta$-spectrin, Futsch, and Fasciclin II levels were unchanged upon knockdown or overexpression of calpain A in postsynaptic muscles by 57 -Gal4. Scale bar, $2 \mu \mathrm{m}$. $\boldsymbol{F}$, Quantitative analysis of fluorescence intensity of synaptic tested proteins in indicated genotypes. $n=8 . p>0.05$ by one-way ANOVA with Tukey post hoc test. Data are mean \pm SEM.

ized at NMJ, we first tested the specificity of calpain antibodies. We found an apparent decrease in the staining intensity of calpain $\mathrm{A}$ at the NMJ of Calp $A^{K G}$ mutants and calpain A RNAi knockdown (Fig. 3A). Consistently, Calp $A^{K G}$ mutants and RNAi knockdown by C57-Gal4 showed $60 \%$ and $85 \%$ decrease of the total protein level, respectively (Fig. $3 C, D)$. The relative calpain A protein level was $40 \pm 5.2 \%(p=0.0005)$ and $15 \pm 1.2 \%(p=$ 0.00001 ) of the WT control in calp $A^{K G 13868}$ homozygous mutant and C57-Gal4-RNAi knockdown, respectively (Fig. 3C,D). Similarly, calp $B^{E Y 17422}$ homozygous mutants showed a 50\% decrease ( $p=0.0001$ ), whereas calpain B RNAi driven by C57-Gal4 exhibited an $84 \%$ decrease $(p=0.00001)$ of calpain B protein level $\left(F_{(2,6)}=505.1, R^{2}=0.9941\right.$, ANOVA; Fig. 3C,D).

We then showed that calpain A immunoreactivity surrounded presynaptic terminals labeled by anti-HRP staining (Fig. $3 A$ ). Moreover, calpain A overlapped and surrounded the postsynaptic marker Dlg, the Drosophila homolog of PSD-95, which labels the subsynaptic reticulum (Fig. 3A). Interestingly, confocal im- aging of synaptic boutons at the third instar larval NMJ demonstrates that calpain A immunoreactivity is located underneath the postsynaptic GluRIIA clusters (Fig. 3B). Calpain B immunoreactivity shows a similar distribution and localizes at the postsynaptic site and muscle (data not shown). In summary, both calpains are enriched at the postsynaptic NMJ.

Previous studies have shown that a large number of proteins are cleaved by calpains in cell cultures (Glading et al., 2002; Franco and Huttenlocher, 2005). To test the specificity of the negative regulation of GluRIIA by calpains at the NMJ, we examined other synaptic proteins to which there were specific antibodies available. The intensities of Dlg, Fasciclin II (FasII), Futsch, dPAK, and spectrins at NMJs were unchanged when calpain A was knocked down or overexpressed in postsynaptic muscles by C57-Gal4 ( $p>0.05$; Fig. $3 E, F)$, demonstrating that calpains specifically and negatively regulate GluRIIA level at NMJ. 


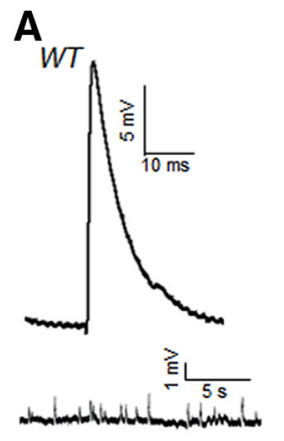

B

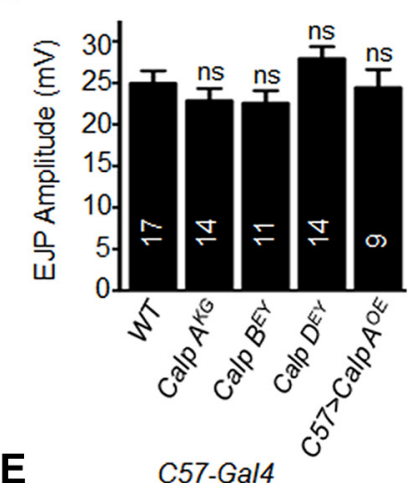

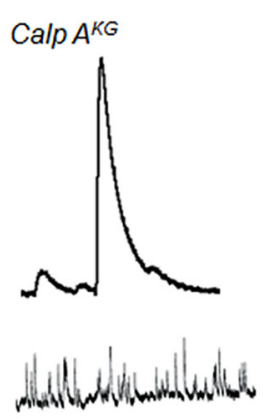

C

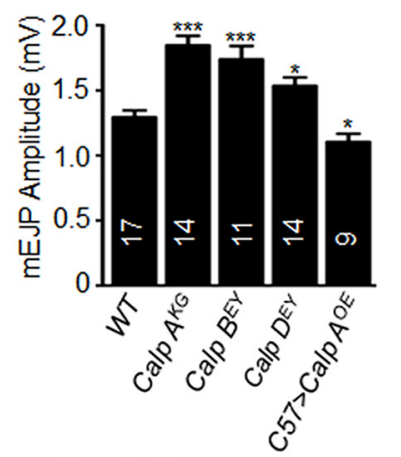

Elav-Gal4

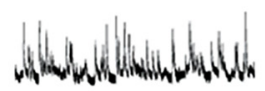

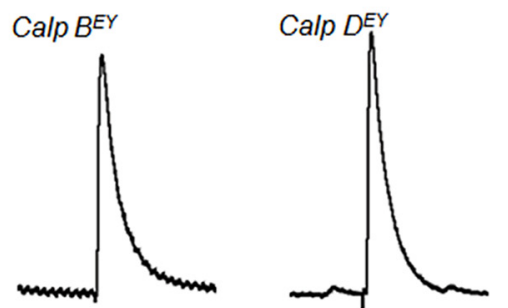

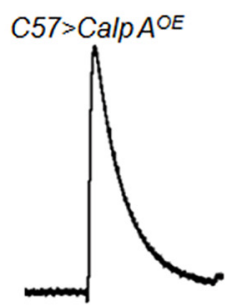

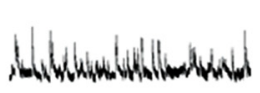

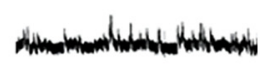

D
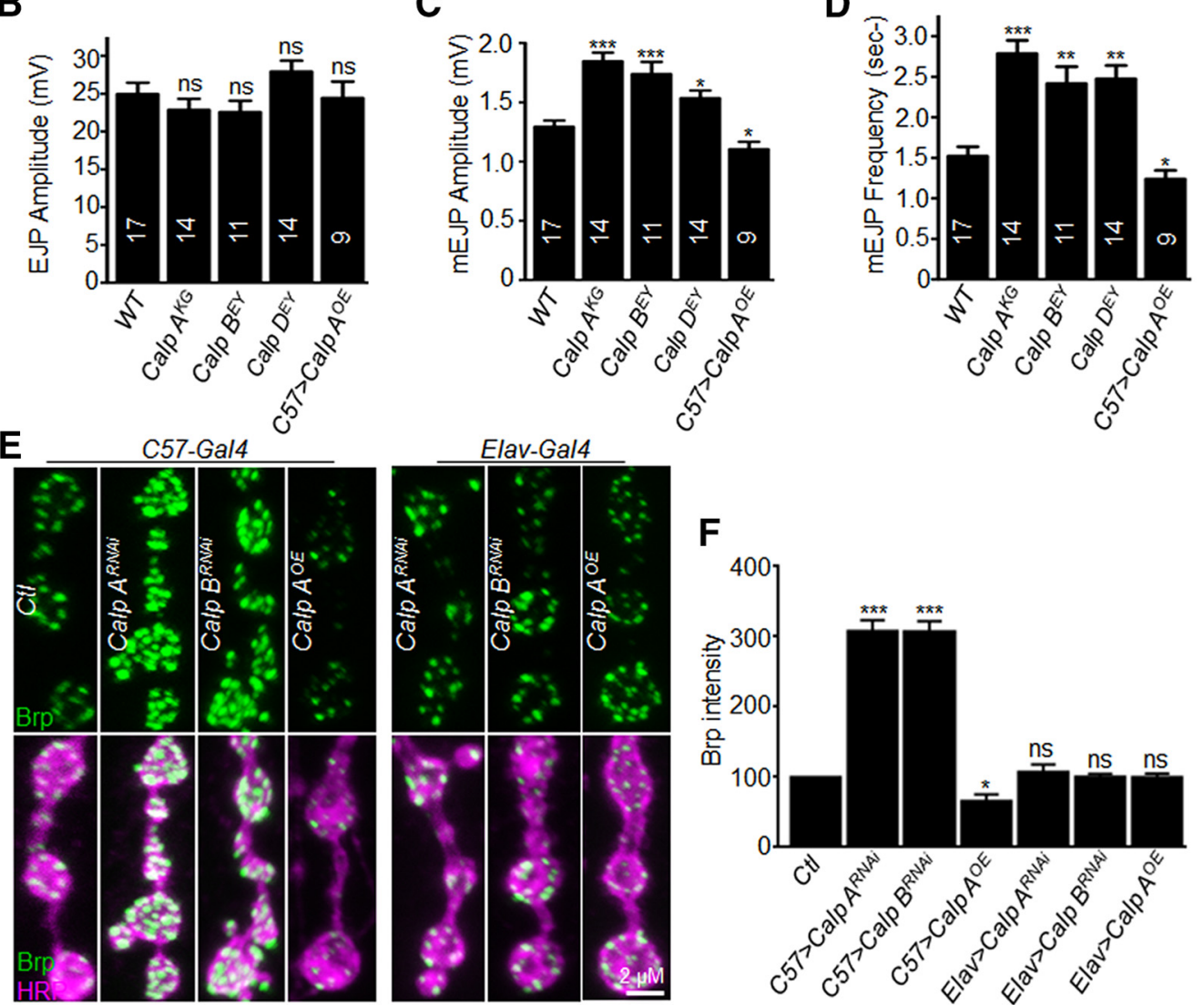

Figure 4. Calpains regulate synaptic function. $\boldsymbol{A}$, Representative recordings of EJPs and mEJPs from different genotypes. $\boldsymbol{B}-\boldsymbol{D}$, Quantitative analysis of EJP amplitudes $(\boldsymbol{B})$, mEJP amplitudes $(\boldsymbol{C})$, and $\mathrm{mEJP}$ frequencies $(\boldsymbol{D})$ from different genotypes. Mean EJP amplitudes were normal, whereas mean $\mathrm{mEJP}$ amplitudes and frequencies in calpain mutants were significantly higher than WT but lower in calpain-overexpressing NMJs. $n=17,14,11,14$, and 9 recordings for WT, calp $A^{K G}$, calp $B^{E Y}$, calp $D^{E Y}$, and calp $A^{O E}$, respectively. ${ }^{*} p<0.05$; ${ }^{* *} p<0.01 ;{ }^{* * *} p<0.001$; one-way ANOVA with Tukey post hoc test. Data are mean \pm SEM. E, Postsynaptic, but not presynaptic, knockdown of calpains gives rise to upregulation of Brp at synapses. Brp intensity is increased upon calpain knockdown, whereas calpain overexpression reduces Brp intensity at NMJ. Representative confocal images of NMJ4 synapses stained with anti-Brp (green) and HRP (magenta) from different genotypes of control, calp $A^{R N A i}$, calp $B^{R N A i}$, and calp $A^{O E}$ under the control of muscle-specific (57-Gal 4 or neuron-specific Elav-Gal4. Scale bar, $2 \mu \mathrm{m}$. $F$, Quantitative analysis of fluorescence intensity of synaptic Brp in indicated genotypes. $n=10$ NMJ terminals for each genotype. ${ }^{*} p<0.05 ;{ }^{* * *} p<0.001$; one-way ANOVA with Tukey post hoc test. Data are mean \pm SEM.

\section{Calpains regulate synaptic function at NMJ}

To determine whether the observed changes in GluRIIA level would affect synaptic transmission in calpain mutants, we recorded EJPs and mEJPs at muscle 6 using intracellular electrodes when calpain expressions were altered (Fig. 4A). We found that the average of EJPs amplitude was normal when calpain expressions were altered $(p>0.05$, ANOVA; Fig. $4 A, B)$. However, we found a significant increase in the average amplitude and frequency of mEJPs in calpain mutants. The average mEJPs was $1.2 \pm 0.05 \mathrm{mV}$ for WT control, $1.9 \pm 0.02$ $\mathrm{mV}$ for Calp $A^{K G}(p=0.00001), 1.8 \pm 0.01 \mathrm{mV}$ for Calp $B^{E Y}$ $(p=0.00002)$, and $1.6 \pm 0.01 \mathrm{mV}$ for Calp $D^{E Y}(p=0.0199)$
(Fig. $4 A, C, D$ ). In contrast, overexpression of calpain A (calp $A^{O E}$ ) led to the opposite; the average mEJPs was $1.0 \pm 0.02 \mathrm{mV}$ for Calp $A^{O E}(p=0.0181$; Fig. $4 A, C, D)$. Consistent with GluRIIA upregulation, the intensity Bruchpilot (Brp), a major constituent of presynaptic active zone characterized by $\mathrm{T}$ bar, was increased when calpains were knocked down in postsynaptic, but not presynaptic, site (Fig. 4E,F). Conversely, calpain overexpression in postsynaptic, but not presynaptic, site resulted in a decrease of Brp intensity (Fig. 4E,F). The increase in GluRIIA and Brp abundance assayed by fluorescence microscopy is positively correlated with increased amplitude and frequency of mEJP at the NMJ synapses. 
A

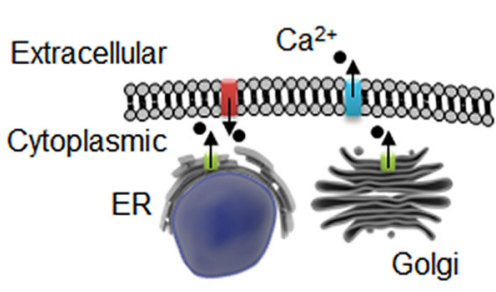

Nck \& PMCA alD \& alT RyR

C

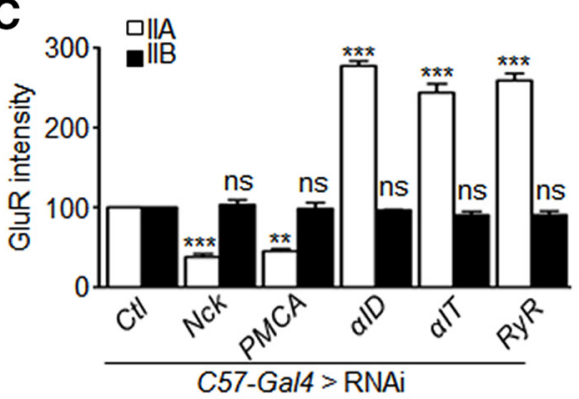

D
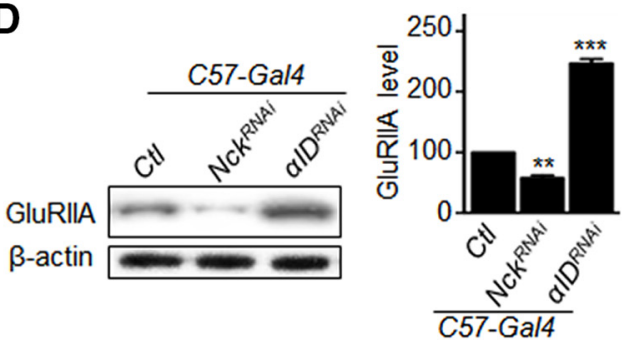

B $\quad$ C57-Gal4 driven muscle specific RNAi
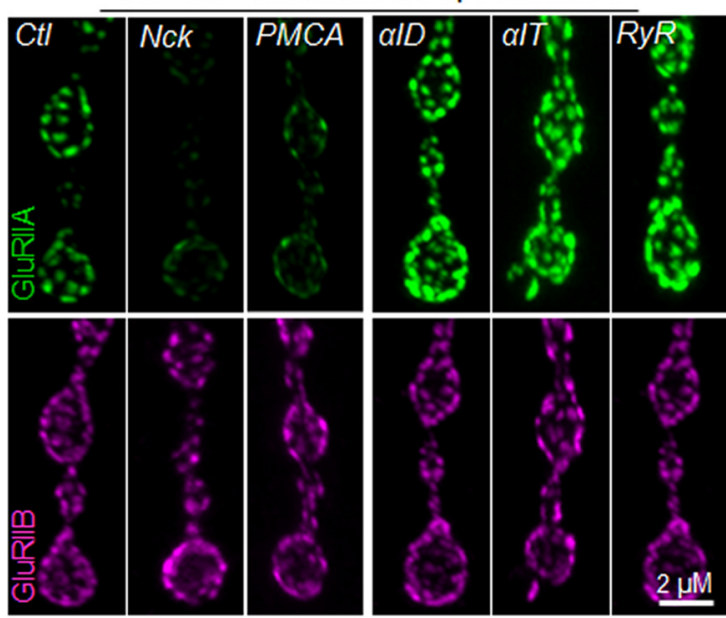

E C57-Gal4 driven muscle specific RNAi
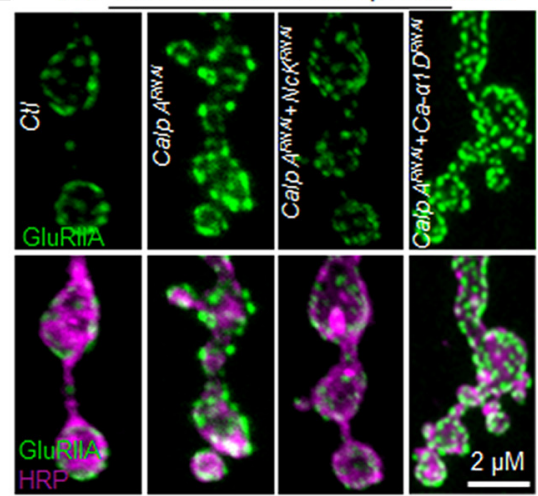

G
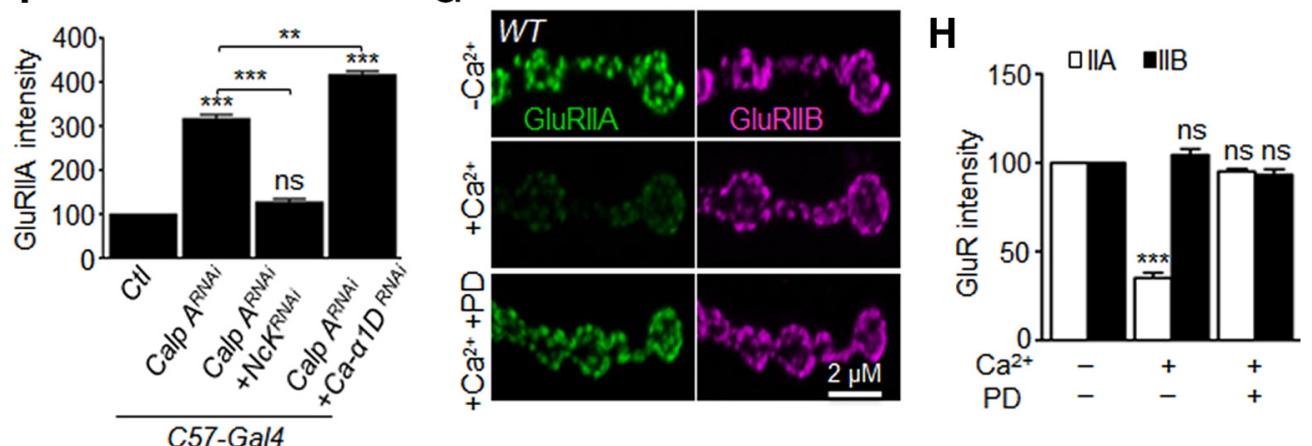

Figure 5. Cytoplasmic calcium negatively regulates GluRIIA level via calpain. $\boldsymbol{A}$, Diagram depicting various calcium channels on different membrane organelles. $\boldsymbol{B}$, Intracellular calcium negatively regulates synaptic GluRIIA. Representative images of NMJ4 synapses from different genotypes costained with anti-GluRIIA (green) and anti-GluRIIB (magenta). Scale bar, $2 \mu$ m. For a list of RNAi lines for $\mathrm{Ca}^{2+}$ channels tested for GluRIIA expression at NMJ, see also Table 5-1 (available at https://doi.org/10.1523/JNEUROSCI.2213-17.2019.t5-1). C, Quantitative analysis of fluorescence intensity of synaptic GluRIIA and GluRIIB in different genotypes. $n=12$ NMJs. ${ }^{* *} p<0.01 ;{ }^{* * *} p<0.001$; one-way ANOVA with Tukey's post hoc test. Data are mean \pm SEM. $D$, Calcium negatively regulates GluRIIA protein levels. Bar graph represents densitometric analysis of the relative levels of GluRIIA. $n=3 .{ }^{* *} p<0.01 ;{ }^{* * *} p<0.001$; one-way ANOVA with Tukey's post hoc test. Data are mean \pm SEM. $\boldsymbol{E}$, Representative confocal images of NMJ4 synapses from indicated genotypes double-stained with anti-GluRIIA (green) and HRP (magenta). Calpain A and calcium channels were knocked down either singly or simultaneously using the muscle-specific (57-Gal4. Scale bar, $2 \mu \mathrm{m}$. $\boldsymbol{F}$, Quantitative analysis of fluorescence intensity of synaptic GluRIIA in different genotypes. $n=12$ NMJs. ${ }^{* *} p<0.01 ;{ }^{* * *} p<0.001$; one-way ANOVA with Tukey's post hoc test. Data are mean \pm SEM. G, Calcium treatment reduced GluRIIA abundance via calpain. Representative images of WT NMJ4 synapses costained with anti-GluRIIA (green) and anti-GluRIIB (magenta). Muscle cells were treated with vehicle dimethylsulfoxide (DMSO), calcium (10 mm), and calcium (10 mm) plus calpain inhibitor PD150606 (20 $\mu \mathrm{m}$ ). Scale bar, $2 \mu \mathrm{m}$. $\boldsymbol{H}$, Quantitative analysis of fluorescence intensity of synaptic GluRIIA and GluRIIB upon calcium treatment. $n=15$ NMJs. ${ }^{* * *} p<0.001$ (one-way ANOVA with Tukey's post hoc test). Data are mean \pm SEM.

Cytoplasmic calcium negatively regulates GluRIIA abundance via calpain

Calcium homeostasis involves an intricate interplay between calcium influx across the plasma membrane and calcium release from the calcium stores, Golgi apparatus, and endoplasmic reticulum (Berridge et al., 2003; Clapham, 2007). As calpains are calcium-dependent proteinases, we hypothesized that calcium might negatively regulate GluRIIA level via calpains. To test the hypothesis, we manipulated cytoplasmic calcium levels by RNAi knockdown of genes encoding calcium channels in the plasma membrane and calcium stores (Fig. 5A; Table 5-1, available at https://doi.org/10.1523/JNEUROSCI.2213-17.2019.t5-1). The 
A

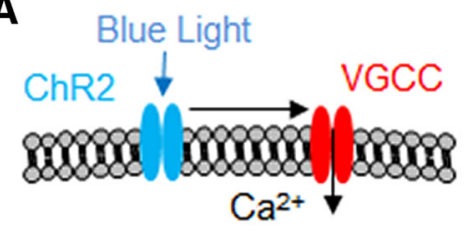

B

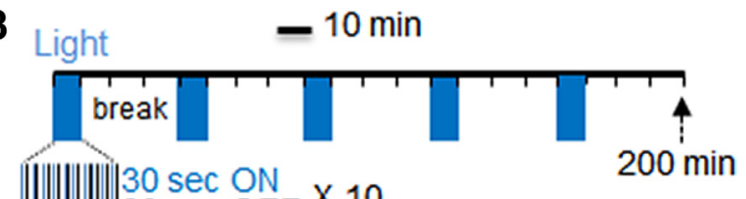

C

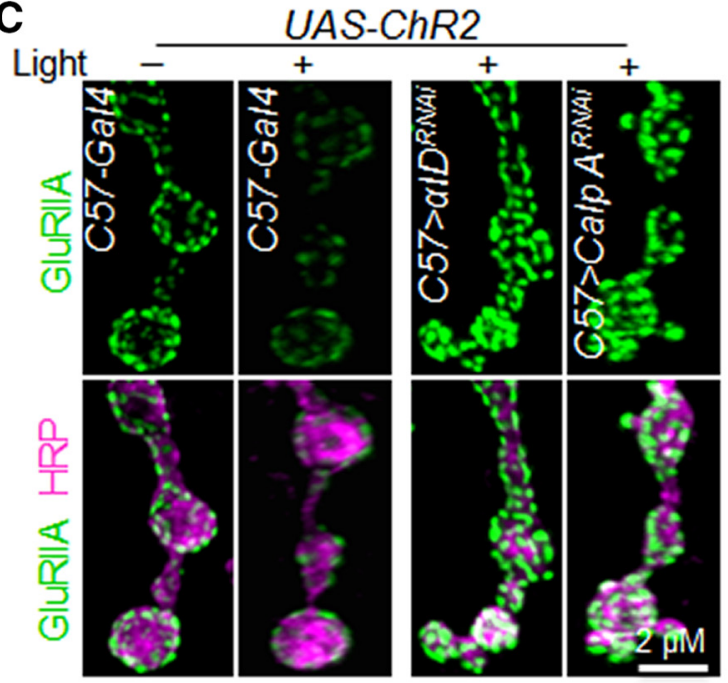

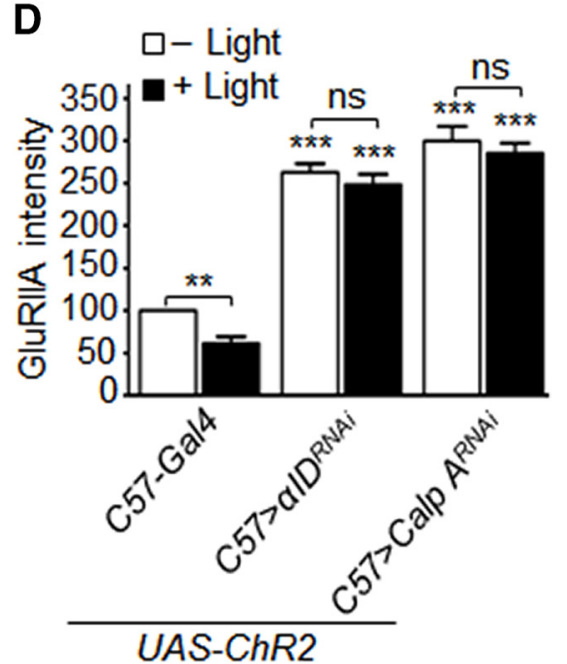

Figure 6. Calcium influx mediated by optogenetic manipulation results in GluRIIA reduction via calpain. A, A schematic of optogenetic manipulation of calcium influx. Blue light activates ChR2, which in turn promotes calcium influx via VGCC. $B$, Light stimulation protocol consists of five rounds of 10 min light stimulation followed by 30 min break. During the illumination period, the light turns on and offfor 30 s each for 10 times. C, Light stimulation resulted in GluRIIA reduction via calpain. Representative images of NMJ4 synapses from different genotypes costained with anti-GluRIIA (green) and anti-HRP (magenta). Scale bar, $2 \mu \mathrm{m}$. D, Quantitative analysis of fluorescence intensity of synaptic GluRIIA in different genotype with or without blue light illumination. $n=15 \mathrm{NMJs}$. ${ }^{* *} p<0.01 ;{ }^{* * *} p<0.001$ (one-way ANOVA with Tukey's post hoc test). Data are mean \pm SEM.

potassium-dependent sodium/calcium exchanger (Nck) and plasma membrane calcium ATPase (PMCA) on the plasma membrane remove intracellular calcium from the cell (HaugCollet et al., 1999; Desai and Lnenicka, 2011), whereas the $\alpha 1 \mathrm{D}$ and $\alpha 1$ T VGCCs (Kanamori et al., 2013) and the ryanodine receptor channels (RyR) increase cytoplasmic calcium by facilitating calcium influx across the plasma membrane and releasing calcium from the calcium stores, respectively (Bi et al., 2014) (Fig. $5 A$ ). Postsynaptic knockdown of both Nck and PMCA channels by C57-Gal4 resulted in a specific and significant reduction in synaptic and total protein levels of GluRIIA but not GluRIIB. In contrast, knockdown of $\alpha 1 \mathrm{D}, \alpha 1 \mathrm{~T}$, and RyR led to the opposite (Fig. $5 B$ ). Compared with control, the relative GluRIIA intensity was $40 \pm 3.4$ for $N c k^{R N A i}(p=0.00001)$ and $50 \pm 3.5$ for $P M C A^{R N A i}(p=0.001)\left(F_{(2,33)}=480.8, R^{2}=0.9866\right.$, ANOVA; Fig. $5 C$ ), whereas the relative intensity of GluRIIA was $280 \pm 4.5$ for $\alpha 1 \mathrm{D}^{\mathrm{RNAi}}(p=0.0002), 250 \pm 3.8$ for $\alpha 1 T^{R N A i}(p=0.0008)$, and $270 \pm 5.6$ for $R y R^{R N A i}(p=0.0004)\left(F_{(3,44)}=119.3, R^{2}=\right.$ 0.8906, ANOVA; Fig. 5C). Different from altered GluRIIA levels, the GluRIIB intensity remained normal $\left(F_{(5,66)}=1.982, R^{2}=\right.$ 0.1305, ANOVA; Fig. $5 B, C)$. Furthermore, to test whether the calcium-regulated GluRIIA level change was mediated through calpains, we examined genetic interaction between calpain $A$ and Nck or $\alpha 1 D$. We found that upregulation of GluRIIA level upon knocking down of calpain A was inhibited by concomitant knockdown of Nck, but not $\alpha 1 \mathrm{D}$ (Fig. $5 E, F$ ). Compared with WT control, the relative GluRIIA intensity was $315 \pm 5.3$ for Calp $A^{R N A i}(p=0.00005), 120 \pm 8.2$ for RNAi knockdown of both calpain A and Nck ( $p=0.3305)$, and $370 \pm 4.5$ for RNAi knockdown of both calpain A and $\alpha 1 \mathrm{D}(p=0.00001)\left(F_{(3,44)}=505\right.$,
$R^{2}=0.9718$; Fig. 5F). Compared with GluRIIA levels in Calp $A^{R N A i}$, the relative GluRIIA intensity was $120 \pm 8.2(p=0.0009)$ for RNAi knockdown of calpain A and NCK and $370 \pm 4.5$ ( $p=$ $0.0013)$ for RNAi of calpain A and $\alpha 1 \mathrm{D}\left(F_{(2,33)}=8.504, R^{2}=\right.$ 0.9551, ANOVA; Fig. $5 F)$. Together, these results indicate that cytoplasmic calcium levels negatively regulate synaptic and total GluRIIA protein levels via calpain.

We further examined the role of calpain in regulating GluRIIA levels by treating larval muscles with the calcium ionophore ionomycin to cause calcium influx (Palmer and Tsien, 2006; Teodoro et al., 2013). Calcium treatment specifically reduced GluRIIA, but not GluRIIB, levels (Fig. 5G,H). The reduction of GluRIIA by calcium was inhibited when the muscles were pretreated with PD150606, a cell-permeable calpain inhibitor (Wang et al., 1996). The relative intensity of GluRIIA was $40 \pm 5.5$ for calciumtreated samples $(p=0.00005)$ and $100 \pm 8.3$ for calpain inhibitor-treated samples $(p=0.2048)$ compared with untreated control $\left(F_{(2,42)}=393, R^{2}=0.9493\right.$, ANOVA; Fig. 5G,H).

These results of genetic and pharmacological analysis together support that calcium negatively regulates GluRIIA, but not GluRIIB, levels via calpains.

\section{Calcium influx mediated by optogenetic manipulations} results in GluRIIA reduction via calpain

To identify for a more physiological condition that promotes calpain activation for GluRIIA cleavage, we designed an experiment to optogenetically induce calcium influx as previously described (Kanamori et al., 2013). We subjected intact and free moving larvae expressing ChR2, a cation channel gated by blue light, to intermittent illumination (Fig. 6A,B) (Honjo et al., 
2012). We found that controlled light exposure resulted in a significant reduction of GluRIIA at NMJ (Fig. $6 C, D$ ); the relative GluRIIA intensity was $50 \pm 4.8$ upon light stimulation $(p=$ $0.008 ; t_{(28)}=27.29$, unpaired $t$ test). Calcium influx to neurons is dependent on voltage-gated calcium channel (VGCC) upon ChR2 activation (Goold and Nicoll, 2010; Kanamori et al., 2013). Consistently, we found that ChR2 activation-induced GluRIIA reduction was dependent on VGCC, as synaptic GluRIIA level was not significantly decreased upon light exposure when $\alpha \mathrm{ID}$, a subunit of VGCC, was knocked down $\left(p=0.3201 ; t_{(28)}=1.054\right.$, unpaired $t$ test; Fig. $6 C, D)$. Furthermore, the observed reduction of GluRIIA upon illumination was inhibited upon calpain A knockdown $\left(p=0.2304 ; t_{(28)}=1.095\right.$, unpaired $t$ test; Fig. $\left.6 C, D\right)$. These results indicate that light-induced synaptic GluRIIA reduction is calcium- and calpain-dependent. Of note, blue light stimulation did not significantly decrease synaptic GluRIIA level compared with untreated controls when $\alpha 1 \mathrm{D}$ or calpain A was knocked down (Fig. 6D). These results together indicate that calcium-induced activation of calpains is likely to occur under certain physiological conditions.

\section{Calcium-dependent cleavage and interaction of GluRIIA with calpain}

The negative regulation of GluRIIA by calpain does not necessarily indicate that calpain cleaves GluRIIA directly. To detect possible GluRIIA truncation by calpains, we expressed N-terminal Myc-tagged GluRIIA and GluRIIB in muscles with the myosin heavy chain promoter. Muscle preparations were treated with calcium, and the muscle lysates were immunoprecipitated with anti-Myc. The intensity of the $120 \mathrm{kDa}$ band representing fulllength GluRIIA showed no apparent change following calcium treatment, while an additional band of $\sim 105 \mathrm{kDa}$ appeared. The $105 \mathrm{kDa}$ band was recognized by anti-Myc, which recognizes the $\mathrm{N}$-terminal tag, and is therefore expected to be the $\mathrm{N}$-terminal large part of GluRIIA (Fig. 7A). Pretreatment of muscles with the calpain inhibitor PD150606 blocked calcium-induced truncation of GluRIIA (Fig. 7A). To test the specificity of the calcium-induced truncation of GluRIIA, we probed immunoblots of anti-Myc immunoprecipitates from muscle lysates expressing Myc-tagged GluRIIB with anti-Myc antibody and found no truncation of GluRIIB upon calcium treatment (Fig. $7 B$ ), demonstrating specific cleavage of GluRIIA at its cytoplasmic C-terminal by calpains.

To verify the calcium-dependent cleavage of GluRIIA by calpains, we performed similar experiments in S2 cells. S2 cells expressing Flag-GluRIIA were lysed and subjected to Western analysis with anti-Flag (Fig. 7C). The intensity of Flag-GluRIIA appeared constant following calcium treatment, but a cleaved GluRIIA fragment of $105 \mathrm{kDa}$ appeared. The intensity of the GluRIIA fragment increased with increasing calcium concentrations from 1 to $10 \mathrm{~mm}$. Compared with $0.1 \pm 0.05$ arbitrary units (a.u.) for untreated controls, calcium treatment at $10 \mathrm{~mm}$ led to a significantly increased intensity of GluRIIA fragment intensity at $0.8 \pm 0.08$ a.u. $\left(p=0.00002, R^{2}=0.9893\right.$; Fig. $\left.7 C, D\right)$, whereas calpain inhibitor treatment reversed the effect of calcium treatment to WT level $\left(p=0.1174 ; F_{(4,10)}=53.54\right.$, ANOVA). The molecular weight of the cleaved GluRIIA fragment was $\sim 105 \mathrm{kDa}$ in $\mathrm{S} 2$ cells (Fig. 7C), similar to that seen in vivo (Fig. 7A), suggesting that calcium-dependent truncation of GluRIIA by calpains occurred at similar sites in vivo and in S2 cells (Fig. 7A-C).

Previous studies have shown that domain I of mammalian calpains 1 and 2 contains a short $\mathrm{N}$ terminus that is cleaved by autolysis, either during or following activation (Siman and No- szek, 1988; Cong et al., 1989). To determine whether calpain activation requires autolysis to cleave GluRIIA, we examined calpains A and B fragments in calcium-treated cells by Western analysis. Autolysis of calpain A and calpain B resulted in the appearance of calpain fragments at lower molecular weights. The densities of these autolytic calpain fragments increased with increasing calcium concentrations (Fig. $7 C$ ). The relative intensity of calpain A fragments was $0.1 \pm 0.04$ a.u. for untreated control and $1.3 \pm 0.06$ a.u. for $10 \mathrm{~mm}$ calcium-treated cells $(p=$ $0.00001)$; the autolytic fragments of calpain A was inhibited when the calpain inhibitor was added $\left(p=0.0232 ; F_{(4,10)}=93.89, R^{2}=\right.$ 0.9742; Fig. $7 C, D$ ). These results demonstrate a close parallel between the levels of autolysis and calpain activity, in accordance with previous reports (Jékely and Friedrich, 1999; Park and Emori, 2008).

Because calpain is enriched at the postsynaptic area and cleaves GluRIIA, we examined whether calpain was associated with GluRIIA. S2 cells cotransfected with plasmids encoding Flag-GluRIIA, calp A-His, and calp B-His were treated with or without calcium (Fig. 7E,F). IP of cell lysates with anti-Flag resulted in coimmunoprecipitation of calpain A and calpain B (Fig. $7 E)$. Reciprocally, cell lysates IPed with anti-calp A resulted in coimmunoprecipitation of Flag-tagged GluRIIA (Fig. $7 F$ ). The association of calpain A and calpain B with GluRIIA was specific because IP with a control nonspecific IgG did not show any positive bands (Fig. 7E,F). In line with the notion of calciuminduced GluRIIA cleavage by calpains, calpain-GluRIIA association was found to be calcium-dependent because nontreated samples showed weak or no coimmunoprecipitation band (Fig. $7 E, F)$.

\section{GluRIIA is cleaved by calpain at Q788}

To precisely map the calpain cleavage site, we first predicted calpain cleavage sites in GluRIIA by bioinformatics software. Based on the size of the cleaved GluRIIA fragment, we expected that the calpain cleavage site in GluRIIA could be near V800. Analysis of the peptide from G785 to F840 of GluRIIA with two different calpain cleavage prediction programs CaMPDB (DuVerle et al., 2011) and GPS-CCD 1.0 (Liu et al., 2011) predicted two highscore calpain cleavage sites at Q788 and K791 (Fig. 8A). Cleavage at either site could theoretically result in N-terminal GluRIIA fragment of $105 \mathrm{kDa}$, as shown in Figure 7. The level of cleaved GluRIIA fragments compared with the full-length protein was very low, indicating that only a small fraction of overexpressed GluRIIA was cleaved by the endogenous calpains (Fig. 7C). To increase calpain cleavage efficiency, we followed an established protocol that uses HNE, a chemical that induces calcium overload in the cell (Sahara and Yamashima, 2010). Cell lysates of S2 cells incubated with various concentrations of HNE were electrophoresed and immunoblotted with anti-GluRIIA. One apparent band of $105 \mathrm{kDa}$ representing the N-terminal fragment of GluRIIA was detected (Fig. 8B).

We then performed mutational analysis for the two predicted sites together or individually for calpain sensitivity (Fig. 8C). S2 cells expressing WT GluRIIA, single or double amino acid mutated GluRIIA, were lysed, and the lysates were electrophoresed and immunoblotted with anti-GluRIIA. The WT GluRIIA and K791A mutant GluRIIA, but not Q788A-K791A or Q788A mutant GluRIIA, were cleaved by calpain in the presence of $\mathrm{HNE}$ (Fig. 8C), indicating that Q788 is the calpain cleavage site. 
A

C

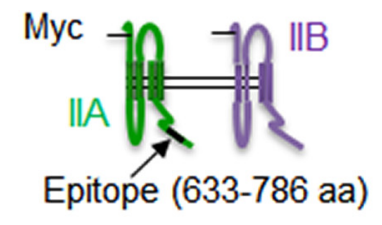

Myc-IIA (IP)

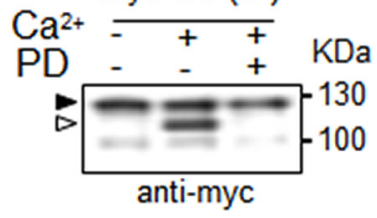

B

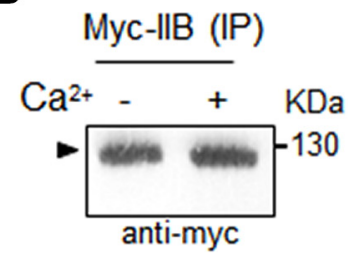

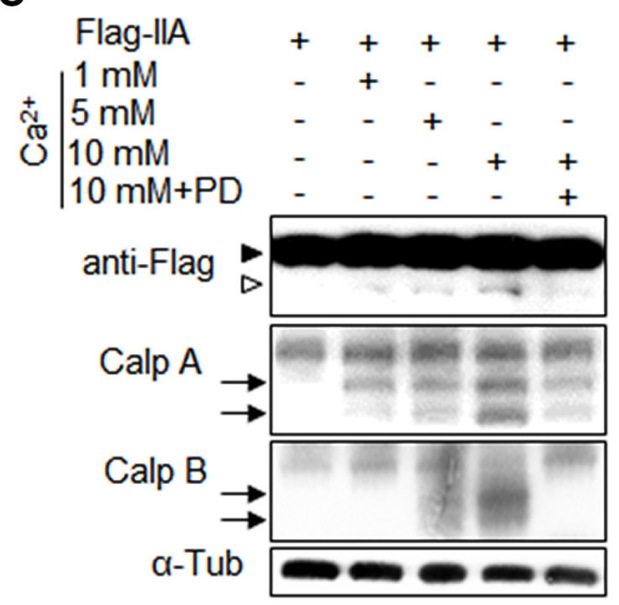
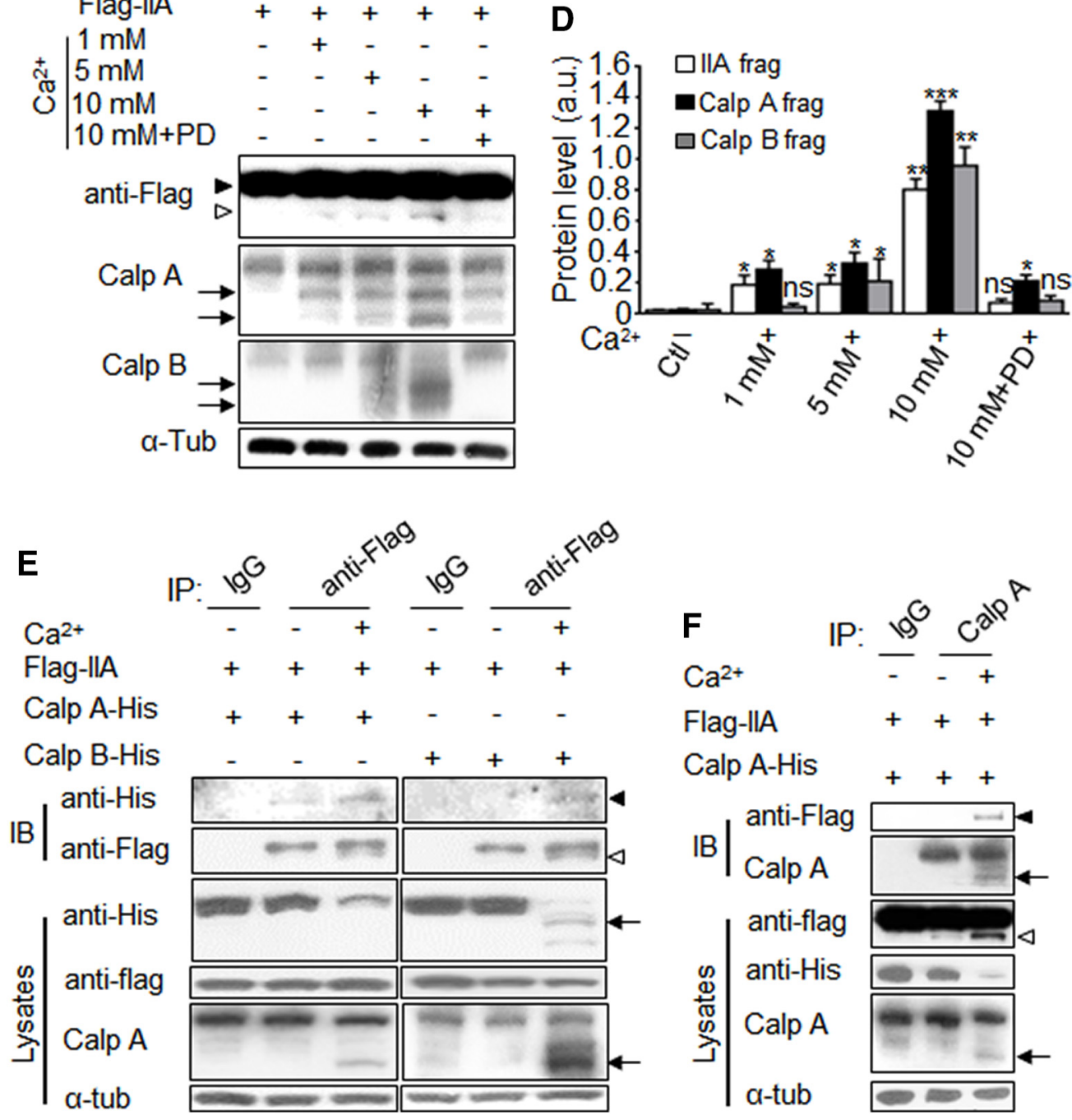

Figure 7. Calcium-dependent cleavage of GluRIIA at C terminus by calpain. $A, B$, Calpain specifically cleaves GluRIIA at its C terminus. Diagram showing location of Myc tags at $\mathrm{N}$ termini. Arrow indicates putative cleavage site. Black bar at the C-terminal of GluRIIIA represents anti-GluRIIA epitope. Muscle cells were treated with vehicle (DMSO), calcium, and calcium plus the calpain inhibitor PD150606. Lysates were subjected to IP with anti-Myc followed by immunoblotting with anti-Myc. Black arrowheads indicate full-length GluRIIA (A) or GluRIIB (B). Open arrowheads indicate truncated N-terminal band of GluRIII. C, Calpain autolysis-associated cleavage of GluRIII. S2 cells expressing Flag-GluRIIA were treated with vehicle (DMSO), calcium at the indicated concentrations, and calcium plus PD150606. Black arrowheads indicate full-length. Open arrowheads indicate N-terminal fragment of GluRIII. Arrows indicate autolytic bands of calpains. $\boldsymbol{D}$, Concentration dependency of calcium-induced truncation of GluRIIIA and calpain autolysis. Quantitative analysis of the level of GluRIIIA, calpain $\mathrm{A}$, and calpain B fragments in arbitrary units (a.u.). $n=3$. ${ }^{*} p<0.05$; ${ }^{* *} p<0.01$; ${ }^{* *} p<0.001$; one-way ANOVA with Tukey's post hoc test. Data are mean \pm SEM. E, F, Calcium-dependent interaction of calpain with GluRIIA. S2 cells coexpressing Flag-IIA and calp A-His or calp B-His were treated with vehicle (DMSO) and calcium $(10 \mathrm{mM})$. Lysates were IPed with anti-Flag antibody or mouse lgG as a control, followed by IB with anti-His and anti-Flag (E). $\boldsymbol{F}$, Lysates were IPed with anti-calp A or rabbit lgG as a control, followed by Western analysis with anti-Flag and anti-calp A. E, $\boldsymbol{F}$, Black arrowheads indicate GluRIIA/calpain interaction. White arrowheads indicate GluRIIA fragments. Arrows indicate calpain autolysis fragments.

\section{Different calpains form a protein complex to} downregulate GluRIIA

As shown in Figure 1, knockdown of different calpains resulted in similar increases of GluRIIA at NMJ synapses, suggesting that they might work synergistically in a similar genetic pathway. To test this possibility, we examined the genetic interactions between different calpains and found that GluRIIA was significantly up- regulated at NMJ synapses in trans-heterozygous mutants for two different calpain genes, whereas mutants heterozygous for a single calpain gene showed normal levels of GluRIIA (Fig. 9A). Compared with the WT, the relative intensity of GluRIIA was unchanged for the heterozygous single calpain gene mutants $\left(p>0.05 ; F_{(3,44)}=0.9460, R^{2}=0.0805\right)$ but significantly upregulated for the trans-heterozygous mutants $(p<0.001$; 
A

\begin{tabular}{|lll|}
\hline 1- CaMPDB & \\
Position & Peptides & Score \\
788 & GELQ|KMKN & 0.23 \\
791 & GELQKMKIN & 0.11 \\
790 & GELQKM|KN & 0.08 \\
796 & WKINH & 0.08 \\
& & \\
\hline
\end{tabular}

\begin{tabular}{|lll|}
\hline 2- GPS-CCD 1.0 & \\
Position & Peptides & Score \\
788 & GELQ|KMKN & 0.812 \\
791 & GELQKMK|NKWW & 0.484 \\
\hline
\end{tabular}

B

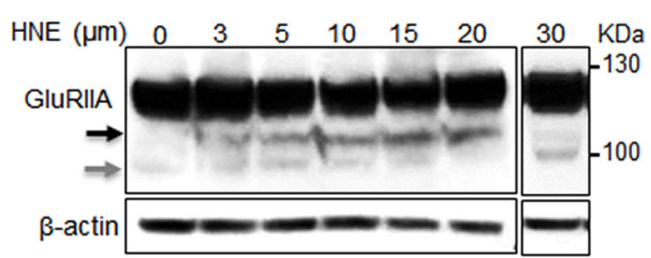

\section{Flag-IIA}

Flag-llA $\left(Q^{788} K^{791}-A A\right) \quad-\quad-\quad+\quad-\quad-$

Flag-llA $\left(K^{791}-A\right)$

Flag-IIA $\left(Q^{788}-A\right)$

HNE $(15 \mu \mathrm{m})$

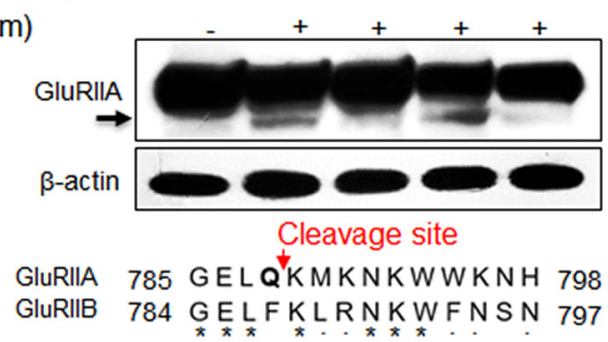

Figure 8. GluRIIA is cleaved by calpain at Q788. A, Bioinformatics analysis of a peptide from $\mathrm{G} 785$ to F840 of GluRIIA for putative calpain cleavage sites. An analysis using CaMPDA (www.calpain. org/prediction_view.rb) showed Q788, M790, K791, and N797 as calpain cleavage sites, whereas an analysis using GPS-CCD 1.0 (http://ccd.biocuck00.org) identified Q788 and K791 as putative calpain cleavage sites. B, HNE induces cleavage of GluRIIIA by calpain in a concentration-dependent manner. S2 cells expressing Flag-GluRIIA were treated with vehicle (DMSO) and HNE at the indicated concentrations. Black arrow indicates the N-terminal fragment of GluRIIA at $105 \mathrm{kDa}$. Gray arrow indicates an unspecific band. $\beta$-Actin was used as a loading control. C, S2 cells expressing WT full-length Flag-IIA, single or double amino acid mutant GluRIIA at Q788 and K791 were analyzed by immunoblotting with anti-GlullA. Black arrow indicates the N-terminal fragments of GluRIIA. $\beta$-Actin was used as a loading control. Red arrow indicates the calpain cleavage site in GluRIIA (based on Accession No. NP_523484), but not GluRIIB (based on Accession No. AAF52269).

$F_{(3,44)}=849.4, R^{2}=0.9830$, ANOVA; Fig. 9B). These results indicate that different calpains might act in the same genetic pathway to downregulate GluRIIA at NMJ synapses.

We speculated that such genetic interactions between calpains may be accomplished via biochemical interactions. To determine whether calpain A physically interacted with calpain B in vivo, we performed coimmunoprecipitation assay. Expression of calpain A-GFP by C57-Gal4 led to GluRIIA downregulation, indicating that it functions as the endogenous calpain A (data not shown). Lysates of muscle samples from calpain A-GFP-expressing larvae were immunoprecipitated with an antibody against GFP. The immunoprecipitates were then subjected to IB analysis with antibodies against calpain B (Fig. 9C). We found that calpain A interacted with calpain B in vivo (Fig. 9C). We noted that calpain A did not form homodimer, given that anti-GFP immunoprecipitated calpain A-GFP only, but not the endogenous calpain A, although both were expressed at comparable levels (Fig. 9C). This is consistent with a previous report that no homodimerization of calpain B was observed (Park and Emori, 2008). Furthermore, a physical interaction between calpains was confirmed in cultured S2 cells. IP of calpain A resulted in coimmunoprecipitation of calpains B-D (Fig. 9D). Similarly, IP of calpain B resulted in coimmunoprecipitation of the other calpains A and C (Fig. 9E). The calpain complex did not appear to be regulated by calcium because the interaction was unchanged by calcium treatment (Fig. 9F).

In support of the physical interactions, we showed that calpain A colocalized with calpain B in discrete puncta within muscle cells (Fig. 9G), and both calpains were enriched at the postsynaptic area. In addition, calpain A was also substantially colocalized with calpains B-D in S2 cells (Fig. 9H).

The observed biochemical interactions between different calpains may somehow affect calpain activity in vivo. However, synaptic and total levels of calpain A and B were not interdependent; the total protein level and synaptic staining of calpain A were unaffected by calpain B or D knockdown (Fig. 10A-D), and calpain $\mathrm{B}$ expression remained normal following calpain A or D knockdown (Fig. 10A-D). For example, compared with WT control, the relative intensity of calpain $A$ in calp $B^{R N A i}$ was $105 \pm 4.5$ $(p=0.9045)$ and the intensity of calpain B in calp $A^{R N A i}$ was $103 \pm 5.4(p=0.9203$; Fig. 10C). We therefore examined the effect of one calpain on the activity of another, using calpain autolysis as a readout. We found that the calcium-induced autolysis sites of calpain A or calpain B were altered, leading to a larger calpain fragment compared with WT when one of the other calpains was mutated (Fig. 10E), suggesting a regulatory role for the calpain complex in calcium-induced autolysis and activation of individual calpains. Our results from genetic and biochemical analyses together support that Drosophila calpains work as a complex (Fig. 10F) to cleave their substrates. Different calpains act together, probably as a protein complex, to control GluR levels during development. In the absence of one calpain, the activity of other calpains is compromised, suggesting that the full activity of calpains requires the integrity of the calpain complex.

\section{Discussion}

Although many studies have focused on the pathological role of calpains, the function of calpain during normal development remains obscure. The data presented here uncover a previously unknown role of calpains at the Drosophila NMJ synapses. First, we present multiple lines of evidence that calpain is enriched at the postsynaptic area and required to downregulate GluR levels in a calcium-dependent manner. Second, we provide genetic and biochemical evidence that different calpains function together, probably as a protein complex. While mammalian calpains act as heterodimers, we reveal in the present study a new mode of calpain activity regulation (i.e., Drosophila calpains work as heteromultimers). 
A

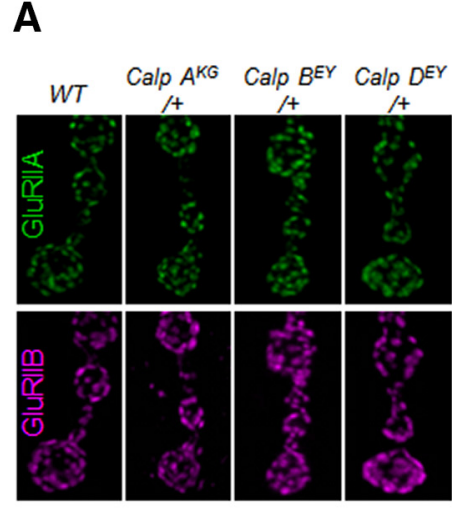

C

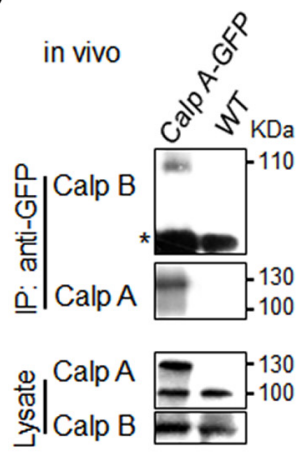

GMuscle expressing Calp A-GFP
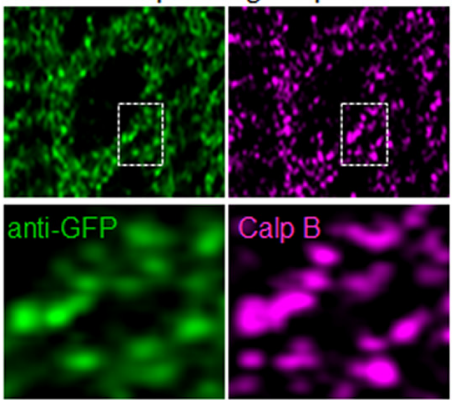

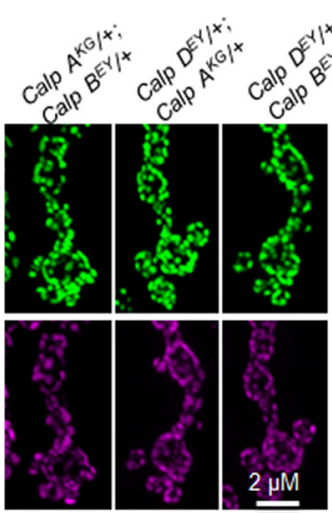

B

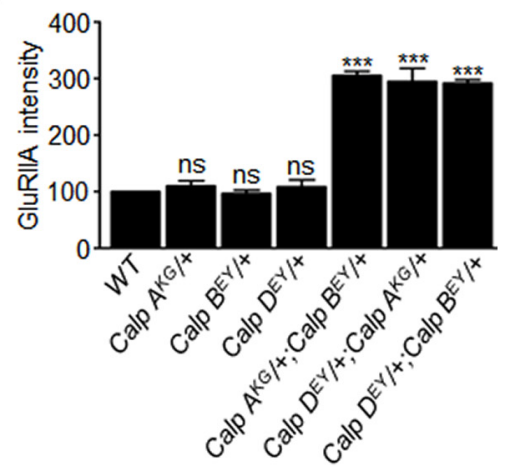

E Calp A-His + -

Calp B-Flag + +

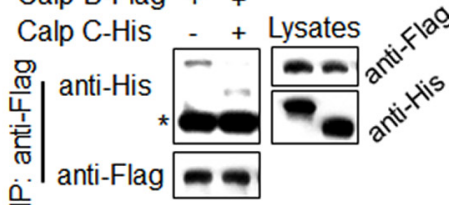

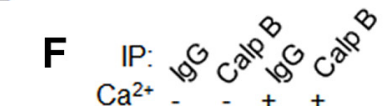

IB:

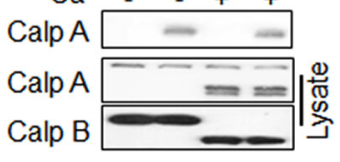

H s2 cells
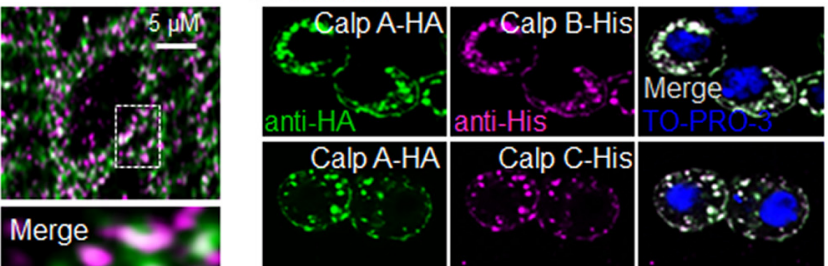

Merge
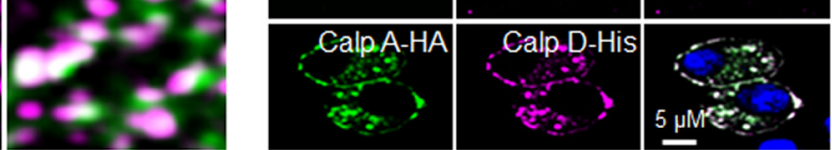

Figure 9. Different calpains form a protein complex to downregulate GluRIIA. $A$, Different calpains interact genetically to downregulate IIA at NMJs. Representative images of NMJ synapses from different genotypes costained with anti-GluRIIA (green) and anti-GluRIIB (magenta). Scale bar, $2 \mu \mathrm{m}$. B, Quantitative analysis of fluorescence intensity of synaptic GluRIIA from indicated genotypes. $n=12 .{ }^{* * *} p<0.001$ (one-way ANOVA with Tukey's post hoc test). Data are mean \pm SEM. C, Calpain A interacts physically with calpain B in vivo. Lysates were extracted from WT and calp A-GFP. Muscle lysates were IPed with anti-GFP and detected with indicated antibodies on Western blots. *IgG heavy chain at $55 \mathrm{kDa}$. D, E, Calpains interact with each other in S2 cells. S2 cells expressing tagged proteins were IPed with anti-calp A (D) or anti-Flag (E), followed by immune-blotting (IB) analysis with the indicated antibodies. ${ }^{*} \lg G$ heavy chain at $55 \mathrm{kDa}$. $\boldsymbol{F}$, Interaction of calpain A and $B$ is not affected by calcium. The lysates were IPed with anti-calp B or rabbit lgG as a control and subsequently detected by Western analysis using the indicated antibodies. $\mathbf{G}$, Representative images of calp A-GFP-expressing muscles costained with anti-GFP (green) and anti-calp B (magenta). Bottom, A higher magnification of the inset. Scale bar, $5 \mu$ m. $\boldsymbol{H}$, HA-tagged calpain A and His-tagged other calpains were substantially colocalized in $\mathrm{S} 2$ cells. S2 cells were stained with anti-HA (green) and anti-His (magenta). Scale bar, $5 \mu \mathrm{m}$.

\section{GluRIIA is specifically cleaved by calpains}

Neurotransmission efficiency is determined by the level of neurotransmitter receptors at the PSDs (Anggono and Huganir, 2012). Therefore, the regulation of glutamate receptors at synapses has been under intensive studies (DiAntonio et al., 1999; Marrus et al., 2004; Schmid et al., 2008). However, the molecular mechanism responsible for regulating the expression of neurotransmitter receptors at synapses is still poorly understood. Using genetic screening, together with optogenetic manipulation and calcium treatment, we revealed that GluRIIA protein levels were specifically and negatively regulated by calcium-dependent calpains during development.

Extensive studies have demonstrated robust homeostatic regulation at the Drosophila NMJ. Genetic or pharmacological manipulations that impair the protein level or activity of postsynaptic GluRs cause a compensatory increase in presynaptic action potential-evoked neurotransmitter release; the increase in evoked release precisely compensates for the decrease in GluR sensitivity and, thereby, maintains a normal level of transmission (Davis and Müller, 2015; Frank, 2014). We showed that calpains negatively regulated mEJP amplitude and frequency at NMJs, consistent with previous studies demonstrating that quantal size and mEJP frequency are increased when GluRIIA is overexpressed (Petersen et al., 1997; DiAntonio et al., 1999; Sigrist et al., 2002). The increase in mEJP amplitude and frequency in calpain mutants can be explained by the elevated levels of GluRIIA and Brp protein at synapses (Figs. 1, 4E,F). GluRIIA mutant exhibits a large decrease in $\mathrm{mEJP}$ amplitude with no change in EJP ampli- 
A

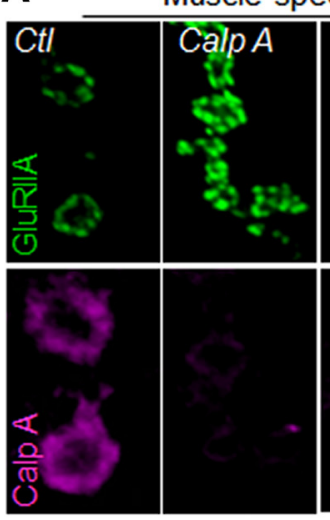

C

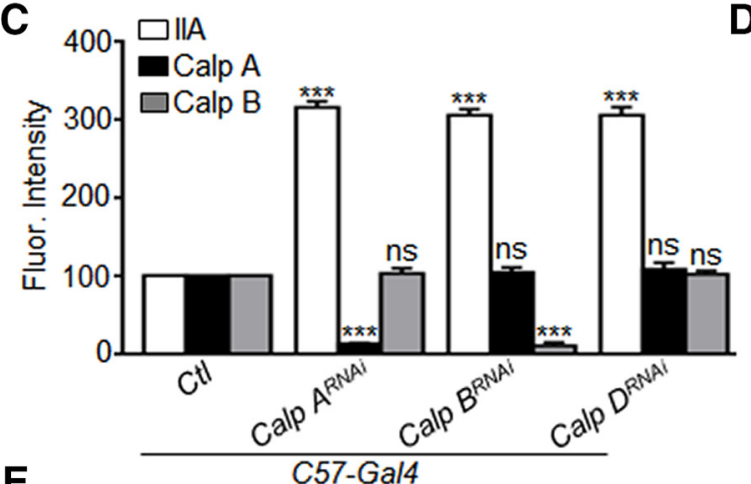

B
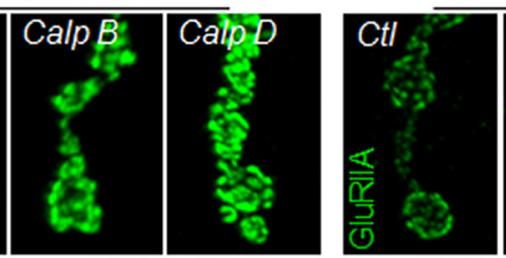

Muscle specific RNAi
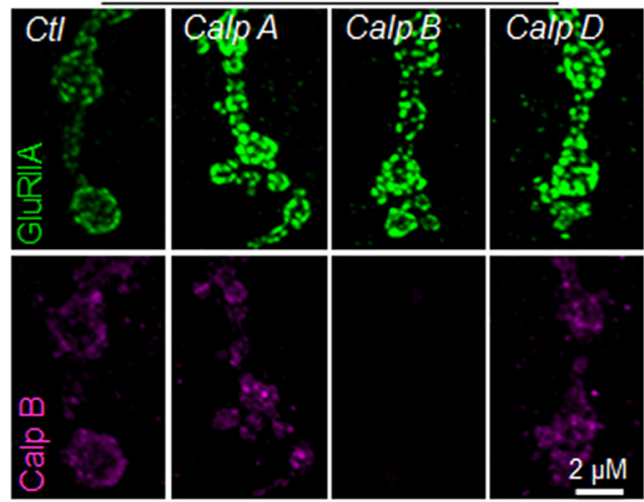

D
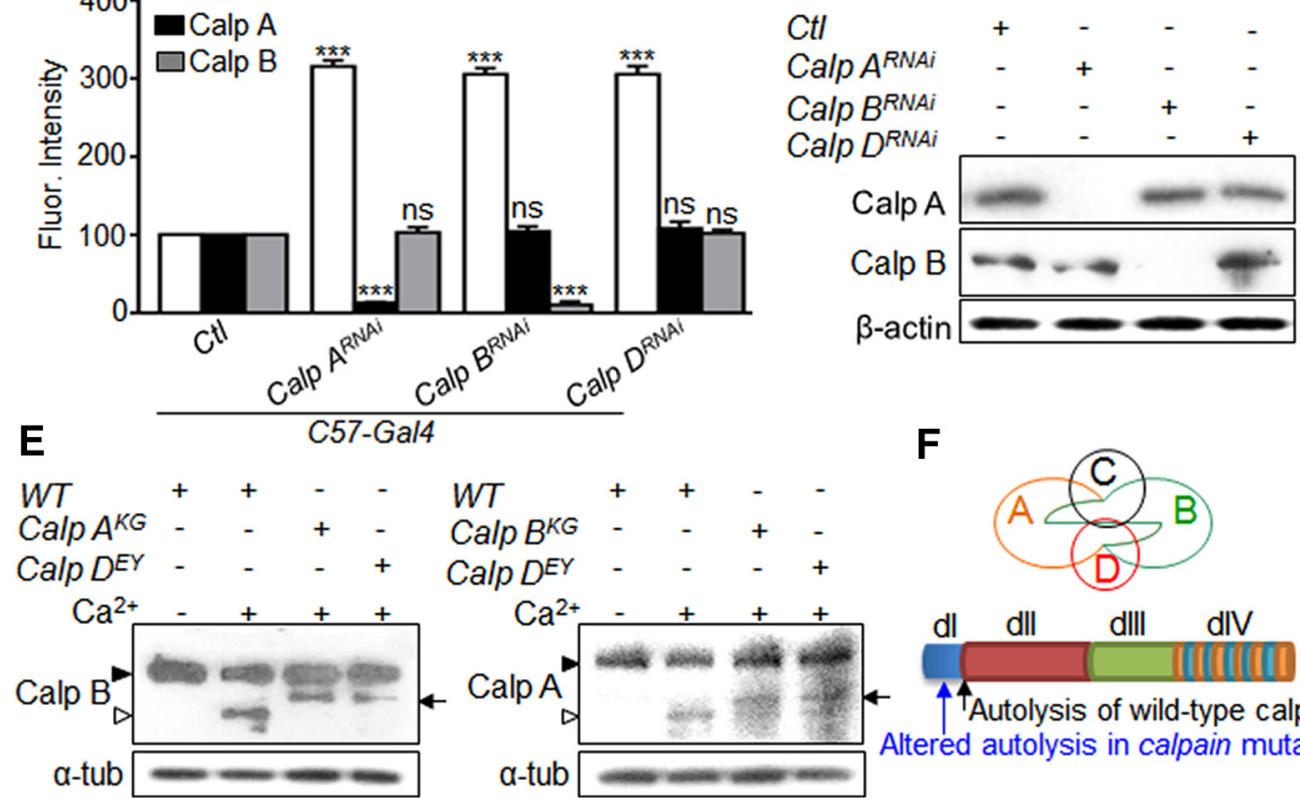

$\mathbf{F}$
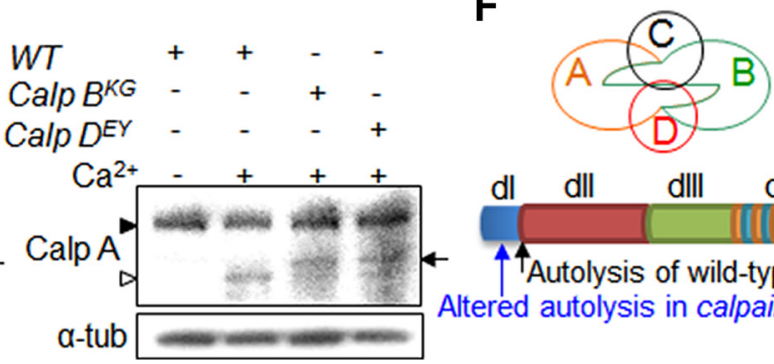

dl dll dIII dIV

个Autolysis of wild-type calpain Altered autolysis in calpain mutants

Figure 10. Calpain activity, but not protein level, depends on the integrity of calpain complex. The expression of calpain $A$ and $B$ is not dependent on each other. $A, B$, Representative images of NMJ4 synapses from indicated genotypes costained with GluRIIA (green) and calp A (magenta) ( $\boldsymbol{A}$ ) or calp B (magenta) (B). Scale bar, $2 \mu \mathrm{m}$. C, Quantitative analysis of fluorescence intensities of synaptic GluRIIA, calpain A, and calpain B in different genotypes. $n=11$ NMJs. ${ }^{* * *} p<0.001$ (one-way ANOVA with Tukey post hoc test). Data are mean \pm SEM. $\boldsymbol{D}$, Representative immunoblots showing the total protein levels of calpains in different genotypes. $\beta$-Actin was used as a loading control. $\boldsymbol{E}$, Muscle lysates from different genotypes were subjected to Western blotting with anti-calp A or anti-calp B. $\alpha$-Tubulin was used as a loading control. Black arrowhead indicates full-length calpain. Open arrowhead indicates autolytic fragments of calpains in WT larvae. Arrows indicate autolytic calpain fragments in calpain mutants. $\boldsymbol{F}$, Different calpains act together, probably as a protein complex, to control GluR levels during development. In the absence of one calpain, the activity of other calpains is compromised, suggesting that the full activity of calpains requires the integrity of the calpain complex.

tude, probably due to homeostatic regulation, whereas overexpression of GluRIIA leads to an increase in mEJP amplitude with an increase of EJP amplitude (Petersen et al., 1997), indicating that there is no compensatory downregulation of quantal content. In the latter case, different effects on EJP amplitude were observed in a more quantitative manner by using a different gene dosage of GluRIIA (DiAntonio et al., 1999). Specifically, increased EJP amplitude is observed at synapse overexpressing a low level of GluRIIA, whereas overexpression of GluRIIA at a high level leads to normal EJP amplitude (DiAntonio et al., 1999). This latter case is consistent with our finding that a high threefold increase of GluRIIA level in calpains mutant did not lead to upregulation of EJP amplitude. It is possible that the increase in mEJP amplitude is compensated by a downregulation of quantal content to maintain normal EJP amplitude in calpain mutants (Fig. 4). Our finding of normal EJP amplitudes regardless of upregulation or downregulation of GluRIIA abundance upon cal- pain manipulations further support homeostatic regulation at Drosophila NMJ.

Previous studies showed that GluRIIA and GluRIIB exhibit competing effects and interdependence as a result of the selective absence of GluRIIA or GluRIIB type at NMJ (DiAntonio et al., 1999; Schmid et al., 2008). If the primary effect of calpains was specifically on GluRIIA, we would expect to see an opposite change in GluRIIB level. However, this is not what we observed. Instead, we observed that GluRIIB was normally localized and its levels were unchanged when GluRIIA was upregulated, resulting from calpain mutation or knockdown, suggesting a distinct regulation of subunit composition by calpains at the NMJ.

At the Drosophila NMJ, the postsynaptic calcium has been speculated to regulate the sensitivity and synaptic localization of GluRIIA (Thomas and Sigrist, 2012). However, how calcium regulates GluRIIA at NMJ is yet to be investigated. Our findings in the present study showed a critical role of calcium in regulating 
postsynaptic GluRIIA levels via calpain. In Drosophila muscles and $\mathrm{S} 2$ cells, calcium-dependent activation of calpain resulted in the $\mathrm{C}$ terminus cleavage of GluRIIA but not GluRIIB. We note that the percentage of cleaved GluRIIA by calpains is low by Western analysis (Figs. 7, 8). This low cleavage efficiency is consistent with previous findings (Bano et al., 2005; Abe and Takeichi, 2007; Xu et al., 2007). However, immunostaining showed apparent changes in GluRIIA level at NMJ upon manipulating calpain expressions (Figs. 1, 2). Multiple possibilities could explain the discrepancy between low cleavage efficiency by Western analysis and apparent changes in GluRIIA level by immunostaining. First, synaptic GluRIIA is part of the total GluRIIA and calpain may preferentially cleave a subset of synaptic GluRIIA, which is physically accessible and sensitive to calpain cleavage due to specific post-translational modifications. The enzymatic activity of calpains could also be differentially regulated at different cellular locations. Alternatively, calpain may indirectly regulate GluRIIA level by other mechanism, such as translation regulation.

There are a few reports that calpain is involved in the regulation of protein synthesis. For example, ischemia-induced calpain activation targets translation initiation factor $4 \mathrm{G} 1$ for cleavage, leading to reduced translation (Vosler et al., 2011). On the other hand, calpain stimulates protein synthesis by truncating B56a, the regulatory subunit of PP2A which inhibits the Akt/mTOR/S6 signaling during mGluR-dependent LTD (Zhu et al., 2017). Although multiple independent lines of evidence support that Drosophila calpains specifically target GluRIIA for cleavage, there is still a possibility that calpain negatively regulates GluRIIA level through other unknown mechanisms, including translational regulation.

Where does GluRIIA get cleaved by calpains within the cell? As calpains are enriched in the postsynaptic area where calcium transients are produced by spontaneous and evoked transmitter release (Desai and Lnenicka, 2011), it is possible that calpain cleaves GluRIIA at the postsynaptic area. Alternatively, calpain might cleave GluRIIA at the endoplasmic reticulum when it is synthesized. As an obvious decrease in GluRIIA intensity was observed upon calcium treatment for a short time of $30 \mathrm{~min}$, we favor the first scenario in which calpain cleaves GluRIIA mostly at the postsynaptic site.

Calpain interacts with and cleaves dozens of target proteins (Franco and Huttenlocher, 2005; Sorimachi et al., 2011). In the present study, we found that calpains specifically target GluRIIA but not other synaptic proteins at the Drosophila NMJ terminals. We further found a physical interaction between calpain and GluRIIA in a calcium-dependent manner. To our knowledge, this is the first demonstration of calcium-facilitated interaction of calpain with its target protein in cultured cells. It will be of interest to test whether other substrates of calpains identified so far also interact with calpains in a calcium-dependent manner.

\section{Calpains are active under physiological conditions}

Calpain activation is triggered by abnormally high calcium levels associated with pathologies, such as ischemic insults and neurodegeneration (Lee et al., 2000; Bano et al., 2005; Xu et al., 2007), but the in vivo role of calpain under physiological conditions is largely unknown. Our genetic and optogenetic results indicate that calcium-induced activation of calpains is likely to occur under certain physiological conditions at NMJ synapses during development. As calcium signaling is involved in a variety of molecular and cellular processes in development and disease, our findings of the present study offer a novel insight into the role of calcium signaling pathways. How and where calpains are activated by calcium within a cell remain to be elucidated.

\section{Different calpains form an active protease complex}

Multiple independent lines of evidence showed that different calpains, at least calpains $\mathrm{A}, \mathrm{B}$, and $\mathrm{D}$, form a complex in which each is essential for the normal proteinase activity to control GluRIIA level. Drosophila calpain B was originally demonstrated to work as monomers based on in vitro studies of Escherichia coliproduced recombinant protein (Park and Emori, 2008), rather than as heterodimers, such as mammalian calpains 1 and 2 (Strobl et al., 2000; Sorimachi et al., 2011). However, our results showed that different calpains acted together in vivo to downregulate GluRIIA. Specifically, we showed that different calpains colocalized and interacted physically in vivo and in cultured cells. Drosophila calpains do not form homodimers, at least for calpain A (this study) and calpain B (Park and Emori, 2008), but instead formed multimers consisting of different calpains, although the domain mediating calpain-calpain interaction remains to be defined. Our finding of a calpain complex is supported by a previous study demonstrating that different calpains in Drosophila function synergistically downstream of calcium transients to trigger dendritic pruning of sensory neurons during metamorphosis (Kanamori et al., 2013). Similarly, mammalian calpains 8 and 9, which are specifically expressed in the gastrointestinal tract, interact physically to form a protein complex called "gastric calpain" involving in gastric mucosal defense (Hata et al., 2010). Thus, in addition to the conventional calpain heterodimers, such as calpains 1 and 2, consisting of a large and a small subunit, heterodimers or heteromultimers of different calpains without a small subunit are emerging as a new mode of calpain activity regulation.

\section{References}

Abe K, Takeichi M (2007) NMDA-receptor activation induces calpainmediated beta-catenin cleavages for triggering gene expression. Neuron 53:387-397.

Albin SD, Davis GW (2004) Coordinating structural and functional synapse development: postsynaptic p21-activated kinase independently specifies glutamate receptor abundance and postsynaptic morphology. J Neurosci 24:6871-6879.

Anggono V, Huganir RL (2012) Regulation of AMPA receptor trafficking and synaptic plasticity. Curr Opin Neurobiol 22:461-469.

Bano D, Young KW, Guerin CJ, Lefeuvre R, Rothwell NJ, Naldini L, Rizzuto R, Carafoli E, Nicotera P (2005) Cleavage of the plasma membrane $\mathrm{Na}^{+} / \mathrm{Ca}^{2+}$ exchanger in excitotoxicity. Cell 120:275-285.

Baudry M, Bi X (2016) Calpain-1 and calpain-2: the yin and yang of synaptic plasticity and neurodegeneration. Trends Neurosci 39:235-245.

Berridge MJ, Bootman MD, Roderick HL (2003) Calcium signalling: dynamics, homeostasis and remodelling. Nat Rev Mol Cell Biol 4:517-529.

Bi J, Wang W, Liu Z, Huang X, Jiang Q, Liu G, Wang Y, Huang X (2014) Seipin promotes adipose tissue fat storage through the ER $\mathrm{Ca}^{2+}$-ATPase SERCA. Cell Metab 19:861-871.

Cao G, Xing J, Xiao X, Liou AK, Gao Y, Yin XM, Clark RS, Graham SH, Chen J (2007) Critical role of calpain I in mitochondrial release of apoptosisinducing factor in ischemic neuronal injury. J Neurosci 27:9278-9293.

Chan SL, Mattson MP (1999) Caspase and calpain substrates: roles in synaptic plasticity and cell death. J Neurosci Res 58:167-190.

Chen K, Merino C, Sigrist SJ, Featherstone DE (2005) The 4.1 protein coracle mediates subunit-selective anchoring of Drosophila glutamate receptors to the postsynaptic actin cytoskeleton. J Neurosci 25:6667-6675.

Clapham DE (2007) Calcium signaling. Cell 131:1047-1058.

Cong J, Goll DE, Peterson AM, Kapprell HP (1989) The role of autolysis in activity of the $\mathrm{Ca}^{2+}$-dependent proteinases (mu-calpain and $\mathrm{m}$-calpain). J Biol Chem 264:10096-10103.

Davis GW, Müller M (2015) Homeostatic control of presynaptic neurotransmitter release. Annu Rev Physiol 77:251-270. 
Davis GW, DiAntonio A, Petersen SA, Goodman CS (1998) Postsynaptic PKA controls quantal size and reveals a retrograde signal that regulates presynaptic transmitter release in Drosophila. Neuron 20:305-315.

Desai SA, Lnenicka GA (2011) Characterization of postsynaptic $\mathrm{Ca}^{2+}$ signals at the Drosophila larval NMJ. J Neurophysiol 106:710-721.

DiAntonio A, Petersen SA, Heckmann M, Goodman CS (1999) Glutamate receptor expression regulates quantal size and quantal content at the Drosophila neuromuscular junction. J Neurosci 19:3023-3032.

Doshi S, Lynch DR (2009) Calpain and the glutamatergic synapse. Front Biosci (Schol Ed) 1:466-476.

DuVerle DA, Ono Y, Sorimachi H, Mamitsuka H (2011) Calpain cleavage prediction using multiple kernel learning. PLoS One 6:e19035.

Fontenele M, Lim B, Oliveira D, Buffolo M, Perlman DH, Schupbach T, Araujo H (2013) Calpain A modulates toll responses by limited Cactus/ IkappaB proteolysis. Mol Biol Cell 24:2966-2980.

Franco SJ, Huttenlocher A (2005) Regulating cell migration: calpains make the cut. J Cell Sci 118:3829-3838.

Frank CA (2014) Homeostatic plasticity at the Drosophila neuromuscular junction. Neuropharmacology 78:63-74.

Friedrich P, Tompa P, Farkas A (2004) The calpain-system of Drosophila melanogaster: coming of age. Bioessays 26:1088-1096.

Glading A, Lauffenburger DA, Wells A (2002) Cutting to the chase: calpain proteases in cell motility. Trends Cell Biol 12:46-54.

Goold CP, Nicoll RA (2010) Single-cell optogenetic excitation drives homeostatic synaptic depression. Neuron 68:512-528.

Hanna RA, Campbell RL, Davies PL (2008) Calcium-bound structure of calpain and its mechanism of inhibition by calpastatin. Nature 456: 409-412.

Hata S, Abe M, Suzuki H, Kitamura F, Toyama-Sorimachi N, Abe K, Sakimura K, Sorimachi H (2010) Calpain 8/nCL-2 and calpain 9/nCL-4 constitute an active protease complex, G-calpain, involved in gastric mucosal defense. PLoS Genet 6:e1001040.

Haug-Collet K, Pearson B, Webel R, Szerencsei RT, Winkfein RJ, Schnetkamp PP, Colley NJ (1999) Cloning and characterization of a potassiumdependent sodium/calcium exchanger in Drosophila. J Cell Biol 147:659-670.

Honjo K, Hwang RY, Tracey WD Jr (2012) Optogenetic manipulation of neural circuits and behavior in Drosophila larvae. Nat Protoc $7: 1470-1478$.

Hood JL, Brooks WH, Roszman TL (2004) Differential compartmentalization of the calpain/calpastatin network with the endoplasmic reticulum and Golgi apparatus. J Biol Chem 279:43126-43135.

Jékely G, Friedrich P (1999) Characterization of two recombinant Drosophila calpains: CALPA and a novel homolog, CALPB. J Biol Chem 274:23893-23900.

Kanamori T, Kanai MI, Dairyo Y, Yasunaga K, Morikawa RK, Emoto K (2013) Compartmentalized calcium transients trigger dendrite pruning in Drosophila sensory neurons. Science 340:1475-1478.

Lee MS, Kwon YT, Li M, Peng J, Friedlander RM, Tsai LH (2000) Neurotoxicity induces cleavage of p35 to p25 by calpain. Nature 405:360-364.

Li W, Yao A, Zhi H, Kaur K, Zhu YC, Jia M, Zhao H, Wang Q, Jin S, Zhao G, Xiong ZQ, Zhang YQ (2016) Angelman syndrome protein Ube3a regulates synaptic growth and endocytosis by inhibiting BMP signaling in Drosophila. PLoS Genet 12:e1006062.

Liu Z, Cao J, Gao X, Ma Q, Ren J, Xue Y (2011) GPS-CCD: a novel computational program for the prediction of calpain cleavage sites. PLoS One 6:e19001.

Marrus SB, Portman SL, Allen MJ, Moffat KG, DiAntonio A (2004) Differential localization of glutamate receptor subunits at the Drosophila neuromuscular junction. J Neurosci 24:1406-1415.

McGuire SE, Le PT, Osborn AJ, Matsumoto K, Davis RL (2003) Spatiotemporal rescue of memory dysfunction in Drosophila. Science 302:17651768 .
Moldoveanu T, Hosfield CM, Lim D, Elce JS, Jia Z, Davies PL (2002) $\mathrm{A} \mathrm{Ca}^{2+}$ switch aligns the active site of calpain. Cell 108:649-660.

Ono Y, Saido TC, Sorimachi H (2016) Calpain research for drug discovery: challenges and potential. Nat Rev Drug Discov 15:854-876.

Palmer AE, Tsien RY (2006) Measuring calcium signaling using genetically targetable fluorescent indicators. Nat Protoc 1:1057-1065.

Park MW, Emori Y (2008) Drosophila calpain B is monomeric and autolyzes intramolecularly. J Biochem 143:217-228.

Petersen SA, Fetter RD, Noordermeer JN, Goodman CS, DiAntonio A (1997) Genetic analysis of glutamate receptors in Drosophila reveals a retrograde signal regulating presynaptic transmitter release. Neuron 19:1237-1248.

Qin G, Schwarz T, Kittel RJ, Schmid A, Rasse TM, Kappei D, Ponimaskin E, Heckmann M, Sigrist SJ (2005) Four different subunits are essential for expressing the synaptic glutamate receptor at neuromuscular junctions of Drosophila. J Neurosci 25:3209-3218.

Reinecke JB, DeVos SL, McGrath JP, Shepard AM, Goncharoff DK, Tait DN, Fleming SR, Vincent MP, Steinhilb ML (2011) Implicating calpain in tau-mediated toxicity in vivo. PLoS One 6:e23865.

Sahara S, Yamashima T (2010) Calpain-mediated Hsp70.1 cleavage in hippocampal CA1 neuronal death. Biochem Biophys Res Commun 393:806-811.

Schmid A, Hallermann S, Kittel RJ, Khorramshahi O, Frölich AM, Quentin C, Rasse TM, Mertel S, Heckmann M, Sigrist SJ (2008) Activity-dependent site-specific changes of glutamate receptor composition in vivo. Nat Neurosci 11:659-666.

Sigrist SJ, Thiel PR, Reiff DF, Schuster CM (2002) The postsynaptic glutamate receptor subunit DGluR-IIA mediates long-term plasticity in Drosophila. J Neurosci 22:7362-7372.

Siman R, Noszek JC (1988) Excitatory amino acids activate calpain I and induce structural protein breakdown in vivo. Neuron 1:279-287.

Sorimachi H, Hata S, Ono Y (2011) Impact of genetic insights into calpain biology. J Biochem 150:23-37.

Strobl S, Fernandez-Catalan C, Braun M, Huber R, Masumoto H, Nakagawa K, Irie A, Sorimachi H, Bourenkow G, Bartunik H Suzuki K, Bode W (2000) The crystal structure of calcium-free human m-calpain suggests an electrostatic switch mechanism for activation by calcium. Proc Natl Acad Sci U S A 97:588-592.

Teodoro RO, Pekkurnaz G, Nasser A, Higashi-Kovtun ME, Balakireva M, McLachlan IG, Camonis J, Schwarz TL (2013) Ral mediates activitydependent growth of postsynaptic membranes via recruitment of the exocyst. EMBO J 32:2039-2055.

Thomas U, Sigrist SJ (2012) Glutamate receptors in synaptic assembly and plasticity: case studies on fly NMJs. Adv Exp Med Biol 970:3-28.

Vosler PS, Gao Y, Brennan CS, Yanagiya A, Gan Y, Cao G, Zhang F, Morley SJ, Sonenberg N, Bennett MV, Chen J (2011) Ischemia-induced calpain activation causes eukaryotic (translation) initiation factor 4G1 (eIF4GI) degradation, protein synthesis inhibition, and neuronal death. Proc Natl Acad Sci U S A 108:18102-18107.

Wang KK, Nath R, Posner A, Raser KJ, Buroker-Kilgore M, Hajimohammadreza I, Probert AW Jr, Marcoux FW, Ye Q, Takano E, Hatanaka M, Maki M, Caner H, Collins JL, Fergus A, Lee KS, Lunney EA, Hays SJ, Yuen P (1996) An alpha-mercaptoacrylic acid derivative is a selective nonpeptide cell-permeable calpain inhibitor and is neuroprotective. Proc Natl Acad Sci U S A 93:6687-6692.

Xu W, Wong TP, Chery N, Gaertner T, Wang YT, Baudry M (2007) Calpain-mediated mGluRlalpha truncation: a key step in excitotoxicity. Neuron 53:399-412.

Zhao G, Wu Y, Du L, Li W, Xiong Y, Yao A, Wang Q, Zhang YQ (2015) Drosophila S6 Kinase like inhibits neuromuscular junction growth by downregulating the BMP receptor thickveins. PLoS Genet 11:e1004984.

Zhu G, Briz V, Seinfeld J, Liu Y, Bi X, Baudry M (2017) Calpain-1 deletion impairs mGluR-dependent LTD and fear memory extinction. Sci Rep $7: 42788$. 